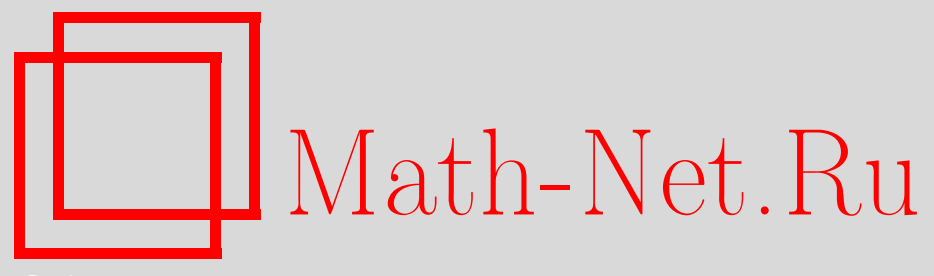

Б. Л. Воронов, Д. М. Гитман, И. В. Тютин, Гамильтониан Дирака со сверхсильным кулоновским полем, ТМФ, 2007, том 150, номер 1, 41-84

DOI: https://doi.org/10.4213/tmf5965

Использование Общероссийского математического портала Math-Net.Ru подразумевает, что вы прочитали и согласны с пользовательским соглашением http://www . mathnet.ru/rus/agreement

Параметры загрузки:

IP : 18.207 .199 .55

26 апреля 2023 г., 15:35:54

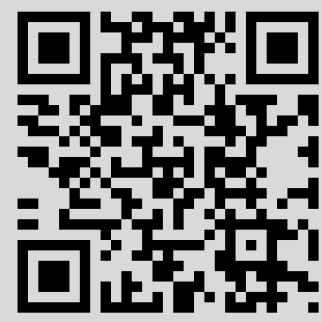




\title{
ГАМИЛЬТОНИАН ДИРАКА СО СВЕРХСИЛЬНЫМ КУЛОНОВСКИМ ПОЛЕМ
}

\begin{abstract}
Рассмотрена проблема квантово-механического описания движения релятивистской дираковской частицы в кулоновском поле точечного заряда $Z e$. В литературе часто высказывается утверждение о том, что квантово-механическое описание такой системы для значений заряда, бо́льших так называемого критического значения с $Z=\alpha^{-1}=137$, не существует. Это мнение основано на том факте, что стандартное выражение для энергии нижнего уровня дает комплексные значения при сверхкритических значениях заряда. Показано, что с математической точки зрения не существует никаких препятствий для построения самосопряженных гамильтонианов при любых значениях заряда. Более того, переход через критическое значение заряда не приводит ни к каким качественным изменениям в математическом описании системы. Специфическая особенность сверхкритических зарядов состоит в том, что самосопряженные гамильтонианы в этом случае строятся не единственным образом. Однако такая неоднозначность существует и при значениях заряда, меньших критического (но бо́льших субкритического значения с $\left.Z=(\sqrt{3} / 2) \alpha^{-1}=118\right)$. Найдены спектры и (обобщенные) собственные функции для всех самосопряженных гамильтонианов. При построении гамильтонианов и их спектральном анализе используются соответственно методы теории самосопряженных расширений симметрических операторов и метод направляющих функционалов Крейна. Вопрос об отношении построенной одночастичной квантовой механики к реальной физике электронов в сверхкритических кулоновских полях, где многочастичные эффекты могут оказаться определяющими, остается открытым.
\end{abstract}

Ключевые слова: дираковский гамильтониан, кулоновское поле, самосопряженные расширения, спектральный анализ.

\section{1. ВВЕДЕНИЕ}

Хорошо известно, что полные системы решений релятивистских волновых уравнений (уравнений Клейна-Гордона, Дирака и т.д.), будучи использованы при квантовании соответствующих свободных полей (скалярного, спинорного и т.д.), позволяют интерпретировать такие квантовые теории в терминах невзаимодействующих

* Физический Институт им. П. Н. Лебедева РАН, Москва, Россия. E-mail: voronov@lpi.ru, tyutin@lpi.ru

${ }^{\dagger}$ Instituto de Física, Universidade de São Paulo, Sao Paulo, Brazil. E-mail: gitman@dfn.if.usp.br 
частиц и античастиц (см., например, [1]). При этом пространство состояний любого такого поля расщепляется на сектора, каждый из которых содержит определенное число частиц (вакуум, одночастичный сектор и т.д.), инвариантное относительно эволюции системы во времени. Описание одночастичного сектора свободной квантовой теории поля может формулироваться как релятивистская квантовая механика, где роль уравнения Шредингера играют соответствующие релятивистские волновые уравнения, а их решения интерпретируются как волновые функции частиц и античастиц. В квантовой электродинамике (КЭД) и в некоторых других моделях часто и плодотворно используется концепция внешнего электромагнитного поля. Одна из точек зрения заключается в том, что теория с внешним полем соответствует приближению, в котором интенсивная часть электромагнитного поля описывается классически и не подвержена обратному влиянию остальной части системы. В КЭД с внешним полем уравнение Дирака с этим полем играет важную роль. Особенно интересные возможности открываются в том случае, когда удается найти точные решения этого уравнения. Известно сравнительно небольшое число физически интересных внешних полей, для которых это возможно [2]. Эти поля можно разделить на группы так, что внутри каждой группы соответствующее уравнение Дирака имеет сходную интерпретацию.

Постоянное и однородное магнитное поле, поле плоской волны и их параллельная суперпозиция принадлежат к группе полей, не нарушающих стабильность вакуума, т.е. не рождающих частицы из вакуума. Точные решения уравнения Дирака с такими полями образуют полные системы, использующиеся в процедуре квантования и позволяющие сохранить интерпретацию квантового спинорного поля во внешнем поле в терминах частиц и античастиц. В КЭД это позволяет построить нулевое приближение, в котором взаимодействие с внешним полем учтено точно, а радиационное взаимодействие с квантованным электромагнитным полем трактуется как возмущение. Такой подход к КЭД с внешним полем известен под названием картины Фарри [3], [1]. В картине Фарри пространство состояний квантовой теории спинорного поля во внешнем поле расщепляется на сектора, каждый из которых содержит определенное число частиц, инвариантное относительно эволюции системы во времени, аналогично случаю квантованного свободного поля. Здесь также оказывается возможным переформулировать теорию в одночастичном секторе как релятивистскую квантовую механику [4]. Следует отметить, что квантовая теория синхротронного излучения полностью базируется на решениях уравнения Дирака с внешним магнитным полем [5], а решения в поле плоской волны широко используются для изучения квантовых эффектов при движении электрона и других частиц спина $1 / 2$ в лазерных пучках [6].

Однородное электрическое поле и некоторые другие электромагнитные поля нарушают стабильность вакуума. Буквальное применение описанного выше подхода для построения картины Фарри в КЭД с такими полями оказывается невозможным. Однако было показано, что существующие точные решения уравнения Дирака с полями электрического типа образуют полные системы функций и могут быть использованы для описания различных квантовых эффектов в таких полях, в частности, для описания эффекта рождения электрон-позитронных пар из вакуума [7]. 
Более того, эти наборы решений позволяют построить обобщение картины Фарри в КЭД с полями, нарушающими стабильность вакуума [8]. Здесь следует отметить, что одночастичный сектор во внешних полях такого типа не стабилен относительно временно́й эволюции, так что соответствующая квантовая механика спиновой частицы в принципе не может быть построена.

Изучение уравнения Дирака с сингулярным внешним полем Ааронова-Бома и с некоторыми дополнительными полями показало, что здесь появляются трудности нового типа. Несмотря на то что в полях указанного типа можно найти некоторые наборы точных решений уравнения Дирака, возникает проблема полноты этих наборов. Эта проблема связана с тем обстоятельством, что гамильтониан Дирака с сингулярным внешним полем Ааронова-Бома требует дополнительного доопределения для того, чтобы его можно было трактовать как самосопряженный (с.с.) квантовомеханический оператор. Можно показать (см., например, обзорную статью [9]), что в этом случае существует целое семейство с.с. гамильтонианов, которые строятся методами теории с.с. расширений симметрических операторов, восходящей к фон Нейману [10]. Каждый с.с. гамильтониан порождает полную систему решений, которую можно использовать для построения картины Фарри в КЭД с сингулярным внешним полем Ааронова-Бома (такое поле не нарушает стабильность вакуума).

Уравнение Дирака с внешним кулоновским полем, а также с некоторыми дополнительными полями всегда было предметом особого интереса. Кулоновское поле даже имеет специальное название "микроскопическое внешнее поле", подчеркивающее его качественное отличие от вышеупомянутых внешних полей, называемых иногда “макроскопическими". До последнего времени здесь господствовала следующая точка зрения: уравнение Дирака для электрона с зарядом ${ }^{1)}-e$ во внешнем кулоновском поле, созданном положительным точечным зарядом $Z e$ ядра с атомным номером $^{2)} Z \leqslant 1 / \alpha=137$ решается точно, имеет полную систему решений и позволяет построить согласующуюся с экспериментом релятивистскую теорию атомных спектров [11]. Такое поле не нарушает стабильность вакуума, так что может быть построена картина Фарри, и существует релятивистская квантовая механика спиновой частицы в таком кулоновском поле. Что касается уравнения Дирака с кулоновским полем с $Z>137$, то оно считалось противоречивым и не имеющим физического смысла [12]-[15]. При этом обычно ссылаются на формулу для энергии нижнего уровня $1 S_{1 / 2}$,

$$
E_{1 s}=m c^{2} \sqrt{1-(Z \alpha)^{2}}
$$

которая формально приводит к мнимым собственным значениям гамильтониана Дирака при $Z>137$. Вопрос о непротиворечивости уравнения Дирака в кулоновском поле с $Z>137$ имеет, с одной стороны, теоретический (математический) интерес, а с другой стороны, связан с вопросом об электронной структуре атомов с атомными номерами $Z>1 / \alpha$ и особенно с ядрами со сверхкритическими зарядами $Z e>170 e$. Последний вопрос имеет фундаментальное значение. Действительно, формулировка КЭД не может считаться завершенной до тех пор, пока не будет получен исчерпывающий ответ на этот вопрос. Хотя ядра с такими большими электрическими

\footnotetext{
1) Константа $e=4,803 \times 10^{-10} \mathrm{CGSE}$ - абсолютная величина заряда электрона.

2) Константа $\alpha=e^{2} /(\hbar c)$ - постоянная тонкой структуры.
} 
зарядами вряд ли могут быть синтезированы ${ }^{3)}$, существующие тяжелые ядра могут имитировать при столкновении сверхкритические кулоновские поля. Ядерные силы могут удерживать сталкивающиеся ядра вместе в течение $10^{-19}$ сек. и более. Этого времени вполне достаточно для эффективного воспроизведения экспериментальной ситуации, когда электрон находится в сверхкритическом кулоновском поле [15]. Задача о поведении электрона в сверхкритическом кулоновском поле рассматривалась рядом авторов [15], [16]. Трудности, связанные с мнимым спектром при $Z>137$, объяснялись тем, что для релятивистского электрона сверхкритическое кулоновское поле слишком сингулярно ${ }^{4)}$. Считалось, что этой проблемы можно избежать, если рассмотреть ядро некоторого конечного радиуса $R$. Было показано, что для $Z<170$ и при обрезании кулоновского потенциала на радиусе $R \sim 1.2 \times 10^{-12}$ см уравнение Дирака имеет физически осмысленные решения [17]. Однако при $Z \sim 170$ даже при наличии обрезания появляется новая трудность. А именно, энергия нижнего уровня опускается до верхней границы нижнего континуума $E=-m c^{2}$, и в этом случае, по общему мнению, задача не может более рассматриваться как одночастичная из-за рождения электрон-позитронных пар, которые, в частности, экранируют кулоновский потенциал ядра. В рамках такой концепции были вычислены вероятности рождения частиц в столкновениях тяжелых ионов [15]. K сожалению, в настоящее время экспериментальные условия для проверки соответствующих предсказаний отсутствуют.

В этой статье мы еще раз возвращаемся к вопросу о непротиворечивости уравнения Дирака с кулоновским полем без обрезания при произвольных значениях заряда ядра (при произвольных $Z$ ). Наша точка зрения заключается в том, что вышеупомянутые трудности со спектром при $Z>137$ не появляются, если корректно определить дираковский гамильтониан как с.с. оператор. Мы представляем строгое рассмотрение всех аспектов этой проблемы и полный спектральный анализ модели, основанные на теории самосопряженных расширений симметрических операторов и методе Крейна направляющих функционалов. Показано, что с математической точки зрения не существует трудности в определении дираковского гамильтониана как с.с. оператора при произвольных $Z$. Более того, при переходе от некритической к критической области заряда не происходит качественных изменений в математическом описании системы. Особенностью сверхкритических зарядов является неединственность с.с. дираковского гамильтониана, однако эта неединственность характерна уже для зарядов, меньших критического (но бо́льших субкритического с $\left.Z=(\sqrt{3} / 2) \alpha^{-1}=118\right)$. Представляя строгое рассмотрение задачи, мы также производим сравнение с обычным физическим подходом к построению квантовомеханического описания релятивистской кулоновской системы. Оказывается, что многие математические результаты могут быть получены из физических соображений за исключением важного свойства полноты системы собственных функций гамильтониана. Найденные полные наборы решений могут быть использованы для

3) Максимум, достигнутый к настоящему времени, есть $Z=118$.

4) Уравнение для радиальных компонент волновой функции имеет форму нерелятивистского уравнения Шредингера с некоторым эффективным потенциалом, который обладает сингулярностью в нуле вида $r^{-2}$, что ассоциируется с падением на центр. 
построения картины Фарри в КЭД. Однако остается неясным, является ли пренебрежение радиационным взаимодействием в такой картине Фарри хорошим нулевым приближением для описания квантовых эффектов в КЭД с кулоновским полем без обрезания при произвольных значениях заряда ядра. Другими словами, остается неясным соответствие построенной квантовой механики с неограниченным снизу спектром энергии реальной физике электрона в сверхкритических кулоновских полях, где многочастичные эффекты могут оказаться определяющими.

Статья организована следующим образом. В разделе 2 мы приводим основные сведения и формулы, поясняющие постановку задачи. Здесь же приводится математически строгая процедура редукции задачи построения с.с. дираковского гамильтониана с внешним кулоновским полем в полном гильбертовом пространстве к задаче построения с.с. одномерных радиальных гамильтонианов. В разделе 3 приведены выражения для общего решения радиальных уравнений, а также некоторые используемые далее частные решения этих уравнений. В пункте 4.1 и приложении А кратко изложены процедуры построения с.с. расширений симметрических дифференциальных операторов и их спектрального анализа. В пунктах 4.2-4.5 построены с.с. дираковские гамильтонианы во всех четырех возможных областях заряда, найдены их спектры и соответствующие полные системы собственных функций.

\section{2. ПОСТАНОВКА ЗАДАЧИ}

Мы рассматриваем уравнение Дирака для частицы спина $1 / 2$ и заряда $q_{1}$, движущейся во внешнем кулоновском поле заряда $q_{2}$; для электрона в водородоподобном атоме $q_{1}=-e, q_{2}=Z e$. Электромагнитный потенциал выбирается в виде

$$
A_{0}=\frac{q_{2}}{r}, \quad A_{k}=0 .
$$

Уравнение Дирака с таким полем, записанное в форме уравнения Шредингера, имеет вид ${ }^{5)}$

$$
i \frac{\partial \Psi(x)}{\partial t}=\check{H} \Psi(x), \quad x=\left(x^{0}, x^{k}\right)=(t, \mathbf{r}),
$$

где $\Psi(x)=\left\{\psi_{\alpha}(x)\right\}$ есть биспинор; дираковский гамильтониан $\check{H}$ задается формулой

$$
\check{H}=\boldsymbol{\alpha} \check{\mathbf{p}}+m \beta-\frac{q}{r}=\left(\begin{array}{cc}
m-q / r & \boldsymbol{\sigma} \check{\mathbf{p}} \\
\boldsymbol{\sigma} \check{\mathbf{p}} & -m-q / r
\end{array}\right),
$$

$m$ есть масса фермиона, $\check{\mathbf{p}}=\left(\check{p}^{k}=-i \partial_{k}\right)$ и $q=-q_{1} q_{2}$; для электрона в водородоподобном атоме $q=Z \alpha$. Для краткости мы далее будем называть константу связи $q$ зарядом. Мы ограничимся случаем $q>0$, поскольку результаты для случая $q<0$ могут быть получены с помощью преобразования зарядового сопряжения.

5) Жирные символы используются для обозначения 3-векторов, для $\gamma$-матриц используется стандартное представление,

$$
\boldsymbol{\alpha}=\left(\begin{array}{cc}
0 & \boldsymbol{\sigma} \\
\boldsymbol{\sigma} & 0
\end{array}\right), \quad \beta=\gamma^{0}=\left(\begin{array}{cc}
I & 0 \\
0 & -I
\end{array}\right), \quad \boldsymbol{\Sigma}=\left(\begin{array}{cc}
\boldsymbol{\sigma} & 0 \\
0 & \boldsymbol{\sigma}
\end{array}\right)
$$

$\boldsymbol{\sigma}=\left(\sigma^{k}\right)$ суть матрицы Паули. Используется запись $\boldsymbol{\sigma} \mathbf{p}=\sigma^{k} p^{k}, \boldsymbol{\sigma} \mathbf{r}=\sigma^{k} x^{k}$ и т.п. В дальнейшем мы полагаем $\hbar=c=1$. 
На этой начальной стадии постановки задачи гамильтониан $\check{H}$ и другие операторы рассматриваются как формальные с.с. дифференциальные операторы, или, как мы будем говорить, с.с. дифференциальные выражения6) [18], помечаемые перевернутой шляпкой $\vee$ над соответствующим символом. Они становятся квантовомеханическими операторами после указания их области определения в гильбертовом пространстве $\mathcal{H}$ биспиноров $\Psi(\mathbf{r})$,

$$
\mathcal{H}=\sum_{\alpha=1}^{4} \oplus \mathcal{H}_{\alpha}, \quad \mathcal{H}_{\alpha}=L^{2}\left(\mathbb{R}^{3}\right) .
$$

В этом случае символ $\vee$ заменяется на обычный символ $\wedge$ над той же самой буквой. В дальнейшем мы будем различать дифференциальные выражения $\check{f}$ и операторы $\hat{f}$ и будем называть $\hat{f}$ оператором, ассоциированным с дифференциальным выражением $\check{f}$.

Цель этой статьи заключается в построении с.с. гамильтониана $\widehat{H}$, ассоциированного с $\check{H}$, что прежде всего означает указание области определения $\widehat{H}$, а в последующем - нахождение его спектра и собственных функций.

Отметим, что в физической литературе проблема собственных значений обычно рассматривается непосредственно в терминах с.с. дифференциального выражения $\check{H}$ как задача на собственные значения дифференциального уравнения $\check{H} \Psi_{E}(\mathbf{r})=$ $E \Psi_{E}(\mathbf{r})$ (стационарного уравнения Дирака) без каких-либо ссылок на область определения гамильтониана ${ }^{7)}$. Оно решается методом разделения переменных, использующим вращательную симметрию задачи. Вращательная симметрия обычно описывается в терминах с.с. дифференциальных выражений следующим образом.

Дираковский гамильтониан $\check{H}$ формально коммутирует с полным угловым моментом $\check{\mathbf{J}}=\check{\mathbf{L}}+\boldsymbol{\Sigma} / 2$, где $\check{\mathbf{L}}=[\mathbf{r} \times \check{\mathbf{p}}]$ есть орбитальный угловой момент, $\boldsymbol{\Sigma} / 2$ есть спиновый угловой момент, а также с так называемым спиновым оператором $\check{K}$,

$$
\check{K}=\beta[1+(\boldsymbol{\Sigma} \check{\mathbf{L}})]=\left(\begin{array}{cc}
\check{\varkappa} & 0 \\
0 & -\check{\varkappa}
\end{array}\right), \quad \check{\varkappa}=1+(\boldsymbol{\sigma} \check{\mathbf{L}}) .
$$

Дифференциальные выражения $\check{H}, \check{\mathbf{J}}^{2}, \check{J}_{3}$ и $\check{K}$ рассматриваются как полный набор коммутирующих операторов, что позволяет выполнить разделение угловых и радиальных переменных и редуцировать полное стационарное уравнение Дирака к радиальному стационарному уравнению Дирака с фиксированными угловым моментом, его проекцией на ось $z$ и собственным значением спинового оператора.

Мы приводим здесь рассмотрение этой проблемы, являющееся строгим с точки зрения функционального анализа. Мы построим с.с. гамильтониан $\widehat{H}$, основываясь на теории с.с. расширений симметрических операторов и на вращательной симметрии задачи. Это означает, что вначале мы определим вращательно-инвариантный симметрический оператор $\widehat{H}^{(0)}$, ассоциированный с с.с. дифференциальным выражением $\check{H}(1)$, что сделать довольно просто, и затем найдем его вращательноинвариантные с.с. расширения. Поскольку коэффициентные функции $\check{H}$ являются

\footnotetext{
6)В математической терминологии - самосопряженные по Лагранжу, в физической терминологии - формально самосопряженные.

7) Фактически некоторая естественная область неявно предполагается.
} 
гладкими вне начала координат, в качестве области определения $D_{H^{(0)}}$ оператора $\widehat{H}^{(0)}$ мы выберем пространство гладких биспиноров с компактным носителем ${ }^{8)}$. Дополнительно потребуем обращения в нуль вблизи начала координат всех биспиноров из $D_{H^{(0)}}$ для того, чтобы обойти проблемы с сингулярностью типа $1 / r$ потенциала ${ }^{9)}$. При этом $\widehat{H}^{(0)}$ определяется следующим образом:

$$
\widehat{H}^{(0)}:\left\{\begin{array}{l}
D_{H^{(0)}}=\left\{\Psi(\mathbf{r}): \psi_{\alpha}(\mathbf{r}) \in D\left(\mathbb{R}^{3}\right) ; \psi_{\alpha}(\mathbf{r})=0, \mathbf{r} \in U_{\varepsilon}\right\}, \\
\widehat{H}^{(0)} \Psi(\mathbf{r})=\check{H} \Psi(\mathbf{r}),
\end{array}\right.
$$

где $D\left(\mathbb{R}^{3}\right)$ - пространство гладких функций в $\mathbb{R}^{3}$ с компактным носителем, а $U_{\varepsilon}$ - некоторая окрестность начала координат, различная, вообе говоря, для разных биспиноров. Область $D_{H^{(0)}}$ плотна в $\mathcal{H}, \overline{D_{H^{(0)}}}=\mathcal{H}$, и интегрированием по частям легко проверяется, что $\widehat{H}^{(0)}$ - симметрический оператор.

Теперь обратимся к вращательной симметрии задачи. Оператор $\widehat{H}^{(0)}$, очевидно, коммутирует с с.с. оператором углового момента $\hat{\mathbf{J}}=\left\{\hat{J}_{k}\right\}$ и с.с. оператором $\widehat{K}$, которые ассоциированы с соответствующими дифференциальными выражениями $\check{\mathbf{J}}$ и $\check{K}$. Операторы $\hat{J}_{k}$ определяются как генераторы унитарного представления группы вращений $\operatorname{Spin}(3)$ в гильбертовом пространстве $\mathcal{H}$. Гильбертово пространство $\mathcal{H}$ представляется в виде прямой ортогональной суммы,

$$
\mathcal{H}=\sum_{j, \zeta}^{\oplus} \mathcal{H}_{j, \zeta}, \quad j=1 / 2,3 / 2, \ldots, \quad \zeta= \pm 1,
$$

подпространств $\mathcal{H}_{j, \zeta}$. Подпространства $\mathcal{H}_{j, \zeta}$ приводят ${ }^{10)}$ операторы $\hat{\mathbf{J}}^{2}, \hat{J}_{k}$ и $\widehat{K}$,

$$
\hat{\mathbf{J}}^{2}=\sum_{j, \zeta}^{\oplus} \hat{\mathbf{J}}_{j, \zeta}^{2}, \quad \hat{J}_{k}=\sum_{j, \zeta}^{\oplus} \hat{J}_{k j, \zeta}, \quad \widehat{K}=\sum_{j, \zeta}^{\oplus} \widehat{K}_{j, \zeta} .
$$

В свою очередь подпространства $\mathcal{H}_{j, \zeta}$ являются конечными прямыми суммами подпространств $\mathcal{H}_{j, M, \zeta}$,

$$
\mathcal{H}_{j, \zeta}=\sum_{M}^{\oplus} \mathcal{H}_{j, M, \zeta}, \quad M=-j,-j+1, \ldots, j .
$$

Подпространство $\mathcal{H}_{j, \zeta, M}$ есть подпространство биспиноров $\Psi_{j, M, \zeta}(\mathbf{r})$ вида

$$
\Psi_{j, M, \zeta}(\mathbf{r})=\frac{1}{r}\left(\begin{array}{c}
\Omega_{j, M, \zeta}(\theta, \varphi) f(r) \\
i \Omega_{j, M,-\zeta}(\theta, \varphi) g(r)
\end{array}\right),
$$

где $\Omega_{j, M, \zeta}(\theta, \varphi)$ - сферические спиноры и $f(r), g(r)$ - радиальные функции (множители $1 / r$ и $i$ введены для удобства). Подпространства $\mathcal{H}_{j, M, \zeta}$ являются собственными для операторов $\hat{\mathbf{J}}^{2}, \hat{J}_{3}$ и $\widehat{K}$,

$$
\begin{aligned}
\hat{\mathbf{J}}^{2} \Psi_{j, M, \zeta}(\mathbf{r}) & =j(j+1) \Psi_{j, M, \zeta}(\mathbf{r}), \quad \hat{J}_{3} \Psi_{j, M, \zeta}(\mathbf{r})=M \Psi_{j, M, \zeta}(\mathbf{r}), \\
\widehat{K} \Psi_{j, M, \zeta}(\mathbf{r}) & =-\zeta(j+1 / 2) \Psi_{j, M, \zeta}(\mathbf{r}),
\end{aligned}
$$

8) Снимая таким образом вопросы, связанные с поведением функций на бесконечности.

9) Строго говоря, мы таким образом оставляем место для $\delta$-образных членов в потенциале.

10)Это означает, что операторы $\hat{\mathbf{J}}^{2}, \hat{J}_{k}$ и $\widehat{K}$ коммутируют с проекторами на подпространства $\mathcal{H}_{j, \zeta}[18]$. 
и очевидно приводят операторы $\hat{\mathbf{J}}^{2}, \hat{J}_{3}$ и $\widehat{K}$. На физическом языке разложение, заданное формулами (2), (3), соответствует разложению биспиноров $\Psi(\mathbf{r}) \in \mathcal{H}$ по собственным функциям коммутирующих операторов $\hat{\mathbf{J}}^{2}, \hat{J}_{3}$ и $\widehat{K}$, что и позволяет произвести разделение переменных в уравнениях для собственных функций.

Отметим, что редукции $\hat{J}_{1,2 j, \zeta}$ операторов $\hat{J}_{1,2}$ на подпространства $\mathcal{H}_{j, \zeta}$ являются ограниченными операторами.

В дальнейшем для нас существенным является следующий факт. Пусть $\mathcal{L}^{2}(0, \infty)$ есть гильбертово пространство дублетов $F(r)$,

$$
F(r)=\left(\begin{array}{c}
f(r) \\
g(r)
\end{array}\right)
$$

со скалярным произведением

$$
\left(F_{1}, F_{2}\right)=\int_{0}^{\infty} F_{1}^{+}(r) F_{2}(r) d r=\int_{0}^{\infty}\left[\overline{f_{1}(r)} f_{2}(r)+\overline{g_{1}(r)} g_{2}(r)\right] d r
$$

так что $\mathcal{L}^{2}(0, \infty)=L^{2}(0, \infty) \oplus L^{2}(0, \infty)$. Тогда формула (4) и соотношение

$$
\left\|\Psi_{j, M, \zeta}\right\|^{2}=\int \Psi_{j, M, \zeta}^{+}(\mathbf{r}) \Psi_{j, M, \zeta}(\mathbf{r}) d \mathbf{r}=\int\left[|f(r)|^{2}+|g(r)|^{2}\right] d r
$$

показывают, что гильбертово пространство $\mathcal{H}_{j, M, \zeta}$ унитарно эквивалентно гильбертову пространству $\mathcal{L}^{2}(0, \infty)$ :

$$
F=U_{j, M, \zeta} \Psi_{j, M, \zeta}, \quad \Psi_{j, M, \zeta}=U_{j, M, \zeta}^{-1} F,
$$

где явная форма унитарного оператора $U$ определяется формулой (4).

Вращательная инвариантность оператора $\widehat{H}^{(0)}$ эквивалентна следующему утверждению.

1. Подпространства $\mathcal{H}_{j, M, \zeta}$ приводят этот оператор, так что $\widehat{H}^{(0)}$ представляется в виде прямой ортогональной суммы своих частей $\widehat{H}_{j, \zeta}^{(0)}$ и $\widehat{H}_{j, M, \zeta}^{(0)}$, которые являются редукциями $\widehat{H}^{(0)}$ на $\mathcal{H}_{j, \zeta}$ и $\mathcal{H}_{j, M, \zeta}$, соответственно,

$$
\widehat{H}^{(0)}=\sum_{j, \zeta}^{\oplus} \widehat{H}_{j, \zeta}^{(0)}, \quad \widehat{H}_{j, \zeta}^{(0)}=\sum_{M}^{\oplus} \widehat{H}_{j, M, \zeta}^{(0)} .
$$

Каждая часть $\widehat{H}_{j, M, \zeta}^{(0)}$ является симметрическим оператором в гильбертовом пространстве $\mathcal{H}_{j, M, \zeta}$. Каждый симметрический оператор $\widehat{H}_{j, M, \zeta}^{(0)}$ в подпространстве $\mathcal{H}_{j, M, \zeta}$ очевидно индуцирует симметрический оператор $\hat{h}_{j, \zeta}^{(0)}$ в гильбертовом пространстве $\mathcal{L}^{2}(0, \infty)$,

$$
\hat{h}_{j, \zeta}^{(0)} F=U_{j, M, \zeta} \widehat{H}_{j, M, \zeta}^{(0)} \Psi_{j, M, \zeta},
$$

так что $\hat{h}_{j, \zeta}^{(0)}=U_{j, M, \zeta} \widehat{H}_{j, M, \zeta}^{(0)} U_{j, M, \zeta}^{-1}$, и $\hat{h}_{j, \zeta}^{(0)}$ задается формулой

$$
\hat{h}_{j, \zeta}^{(0)}:\left\{\begin{array}{l}
D_{h_{j, \zeta}^{(0)}}=\mathcal{D}(0, \infty), \\
\hat{h}_{j, \zeta}^{(0)} F(r)=\check{h}_{j, \zeta} F(r),
\end{array}\right.
$$

где $\mathcal{D}(0, \infty)=D(0, \infty) \oplus D(0, \infty), \quad D(0, \infty)$ - стандартное пространство гладких функций на $(0, \infty)$ с компактным носителем,

$$
D(0, \infty)=\left\{f(r): f(r) \in \mathbb{C}^{\infty}, \operatorname{supp} f \in[a, b], 0<a<b<\infty\right\},
$$


отрезок $[a, b]$ может быть разным для разных $f$; с.с. радиальное дифференциальное выражение $\check{h}_{j, \zeta}$ задается формулой

$$
\check{h}_{j, \zeta}=-i \sigma^{2} \frac{d}{d r}+\frac{\varkappa}{r} \sigma^{1}-\frac{q}{r}+m \sigma^{3},
$$

где $\varkappa=\zeta(j+1 / 2)^{11)}$.

2. Дифференциальное выражение $\check{h}_{j, \zeta}$, а значит, с учетом $(6)$, и оператор $\hat{h}_{j, \zeta}^{(0)}$, не зависят от $M$ при фиксированных $j, \zeta$. Этот факт эквивалентен тому, что оператор $\widehat{H}^{(0)}$ коммутирует с операторами $\hat{J}_{1,2}$, или, более точно, оператор $\widehat{H}_{j, \zeta}^{(0)}$ коммутирует с операторами $\hat{J}_{1,2 j, \zeta}$. На физическом языке $\check{h}_{j, \zeta}$ называется радиальным гамильтонианом, но, строго говоря, радиальный гамильтониан есть с.с. оператор $\hat{h}_{j, \zeta}$, ассоциированный с $\check{h}_{j, \zeta}$.

В дальнейшем под вращательной инвариантностью любого оператора $\hat{f}$ мы будем понимать выполнение следующих требований:

1) приводимость этого оператора подпространствами $\mathcal{H}_{j, M, \zeta}$ и, следовательно, подпространствами $\mathcal{H}_{j, \zeta}$, таким образом, для оператора $\hat{f}$ имеет место формула, аналогичная (5);

2 ) коммутативность его частей $\hat{f}_{j, \zeta}$ с ограниченными операторами $\hat{J}_{1,2 j, \zeta}$ при фиксированных $j, \zeta$.

Пусть $\hat{h}_{j, \zeta}$ есть с.с. расширение симметрического оператора $\hat{h}_{j, \zeta}^{(0)}$ в $\mathcal{L}^{2}(0, \infty)$. Он очевидно порождает с.с. расширения $\widehat{H}_{j, M, \zeta}$ симметрических операторов $\widehat{H}_{j, M, \zeta}^{(0)}$ в подпространствах $\mathcal{H}_{j, M, \zeta}$,

$$
\widehat{H}_{j, M, \zeta}=U_{j, M, \zeta}^{-1} \hat{h}_{j, \zeta} U_{j, M, \zeta}
$$

и при этом оператор $\widehat{H}_{j, \zeta}=\sum_{M}^{\oplus} \widehat{H}_{j, M, \zeta}$ коммутирует с $\hat{J}_{1,2 j \zeta}$. Тогда замыкание прямой суммы

$$
\widehat{H}=\sum_{j, \zeta}^{\oplus} \widehat{H}_{j, \zeta}=\sum_{j, M, \zeta}^{\oplus} \widehat{H}_{j, M, \zeta}
$$

есть с.с. оператор в полном гильбертовом пространстве $\mathcal{H}[19]$, и $\widehat{H}$ является вращательно-инвариантным с.с. расширением исходного вращательно-инвариантного оператора $\widehat{H}^{(0)}$.

Обратно, любое вращательно-инвариантное с.с. расширение $\widehat{H}$ исходного оператора $\widehat{H}^{(0)}$ имеет структуру (9), и при этом оператор $\hat{h}_{j, \zeta}=U_{j, M, \zeta} \widehat{H}_{j, M, \zeta} U_{j, M, \zeta}^{-1}$ в $\mathcal{L}^{2}(0, \infty)$ не зависит от $M$ и является с.с. расширением симметрического оператоpa $\left.\hat{h}_{j, \zeta}^{(0)} 12\right)$.

Задача построения вращательно-инвариантного с.с. гамильтониана $\widehat{H}$ сводится, таким образом, к задаче построения с.с. радиальных гамильтонианов $\hat{h}_{j, \zeta}$.

В дальнейшем мы будем работать при фиксированных $j$ и $\zeta$, поэтому будем опускать эти индексы для краткости. Фактически мы рассматриваем радиальные

11) Отметим, что $\mathcal{D}(0, \infty)$ плотно в $\mathcal{L}^{2}(0, \infty)$, поскольку, как известно, $D(0, \infty)$ плотно в $L^{2}(0, \infty)$, симметричность $\hat{h}_{j, \zeta}^{(0)}$ легко проверяется интегрированием по частям, что подтверждает сделанное выше высказывание.

${ }^{12)}$ Грубо говоря, это означает, что с.с. расширения частей $\widehat{H}_{j, M, \zeta}^{(0)}$ при фиксированных $j$ и $\zeta$ и разных $M$ должны строиться "одинаково". 
дифференциальные выражения $\check{h}_{j, \zeta}$ как единое двухпараметрическое дифференциальное выражение $\check{h}$ с параметрами $q$ и $\varkappa$ (параметры $j$ и $\zeta$ входят через один параметр $\varkappa$, параметр $m$ считается фиксированным) и аналогично рассматриваем ассоциированные радиальные операторы $\hat{h}^{(0)}$ и $\hat{h}$, определенные в одном и том же гильбертовом пространстве $\mathcal{L}^{2}(0, \infty)$.

\section{3. ОБЩЕЕ РЕШЕНИЕ РАДИАЛЬНЫХ УРАВНЕНИЙ}

В дальнейшем нам понадобятся некоторые специальные решения дифференциального уравнения

$$
\breve{h} F=W F,
$$

или, что то же самое, системы уравнений

$$
\begin{aligned}
& \frac{d f}{d r}+\frac{\varkappa}{r} f-\left(W+m+\frac{q}{r}\right) g=0, \\
& \frac{d g}{d r}-\frac{\varkappa}{r} g+\left(W-m+\frac{q}{r}\right) f=0,
\end{aligned}
$$

с произвольным комплексным $W$; вещественные значения $W$ будут обозначаться через $E$, они имеют традиционный смысл энергии. Уравнения (10) мы будем называть радиальными уравнениями. Для полноты изложения мы приведем вывод общего решения радиальных уравнений, следуя стандартной процедуре (см., например, [13], [14]). Сначала представим $f(r)$ и $g(r)$ в виде

$$
f(r)=z^{\Upsilon} e^{-z / 2}[P(z)+Q(z)], \quad g(r)=-i \Lambda z^{\Upsilon} e^{-z / 2}[P(z)-Q(z)],
$$

где $z=-2 i K r, \Upsilon, \Lambda$ и $K$ - некоторые комплексные числа, определяемые ниже. Тогда радиальные уравнения становятся уравнениями для функций $P$ и $Q$. Полагая

$$
\begin{gathered}
\Upsilon^{2}=\varkappa^{2}-q^{2}, \quad \alpha=\Upsilon-i \frac{q W}{K}, \quad \beta=1+2 \Upsilon, \\
W \pm m=\rho_{ \pm} e^{i \varphi_{ \pm}}, \quad 0 \leqslant \varphi_{ \pm}<2 \pi, \\
\Lambda=\sqrt{\frac{W-m}{W+m}}=\sqrt{\frac{\rho_{-}}{\rho_{+}}} e^{i\left(\varphi_{-}-\varphi_{+}\right) / 2}, \quad K=\sqrt{W^{2}-m^{2}}=\sqrt{\rho_{-} \rho_{+}} e^{i\left(\varphi_{-}+\varphi_{+}\right) / 2},
\end{gathered}
$$

мы сводим (10) к системе уравнений

$$
\begin{aligned}
& z \frac{d^{2} Q}{d z^{2}}+(\beta-z) \frac{d Q}{d z}-\alpha Q=0, \\
& P=-\frac{1}{\varkappa-i(q m / K)}\left(z \frac{d}{d z}+\alpha\right) Q .
\end{aligned}
$$

Первое уравнение в (12) есть вырожденное гипергеометрическое уравнение [20], [21] для $Q$.

Пусть $\Upsilon \neq-n / 2^{13)}, n=1,2, \ldots$, тогда общее решение первого из уравнений $(12)$ может быть представлено в виде

$$
Q=A \Phi(\alpha, \beta ; z)+B \Psi(\alpha, \beta ; z),
$$

${ }^{13)}$ Параметр $\Upsilon$ определен в формулах (11) с точностью до знака. Его конкретный выбор определяется соображениями удобства. При соответствующем значении заряда мы будем использовать и $\Upsilon=-n / 2$, этот случай будет ниже рассмотрен отдельно. 
где $A$ и $B$ - произвольные константы, $\Phi(\alpha, \beta ; z)$ и $\Psi(\alpha, \beta ; z)$ - вырожденные гипергеометрические функции, их определение см. в [20], [21] (функция $\Phi(\alpha, \beta ; z)$ не определена при $\beta=0,-1,-2, \ldots)$. Из уравнений (12) и (13) следует, что

$$
P=-\frac{\alpha}{\varkappa-i(q m / K)}\left[A \Phi(\alpha+1, \beta ; z)-B\left(\Upsilon+i \frac{q W}{K}\right) \Psi(\alpha+1, \beta ; z)\right] .
$$

Окончательно общее решение радиальных уравнений (10) для любых комплексных $W$ и действительных $m, \varkappa$ и $q$ дается выражениями

$$
\begin{gathered}
f(r)=z^{\Upsilon} e^{-z / 2}\left\{A\left[\Phi(\alpha, \beta ; z)-a_{+} \Phi(\alpha+1, \beta ; z)\right]+B[\Psi(\alpha, \beta ; z)+b \Psi(\alpha+1, \beta ; z)]\right\}, \\
g(r)=i \Lambda z^{\Upsilon} e^{-z / 2}\left\{A\left[\Phi(\alpha, \beta ; z)+a_{+} \Phi(\alpha+1, \beta ; z)\right]+B[\Psi(\alpha, \beta ; z)-b \Psi(\alpha+1, \beta ; z)]\right\}, \\
a_{ \pm}=\frac{ \pm \Upsilon K-i q W}{\varkappa K-i q m}, \quad b=\frac{\varkappa K+i q m}{K} .
\end{gathered}
$$

С учетом соотношения [20], [21]

$$
\Phi(\alpha+1, \beta ;-2 i K r)=e^{-2 i K r} \Phi(\beta-\alpha-1, \beta ; 2 i K r)
$$

общее решение радиальных уравнений (10) удобно представить в виде

$$
F=\left(\begin{array}{l}
f \\
g
\end{array}\right)=A X(r, \Upsilon, W)+B z^{\Upsilon} e^{-z / 2}\left[\Psi(\alpha, \beta ; z) \vartheta_{+}-b \Psi(\alpha+1, \beta ; z) \vartheta_{-}\right]
$$

где дублеты $\vartheta_{ \pm}$определяются формулой

$$
\vartheta_{ \pm}=\left(\begin{array}{c} 
\pm 1 \\
i \Lambda
\end{array}\right)
$$

а дублет $X$ задается соотношениями

$$
\begin{aligned}
X & =\frac{(m r)^{\Upsilon}}{2}\left[\Phi_{+}(r, \Upsilon, W)+\Phi_{-}(r, \Upsilon, W)\left(\begin{array}{cc}
0 & m+W \\
m-W & 0
\end{array}\right)\right] u_{+}, \\
\Phi_{+} & =e^{i K r} \Phi\left(\Upsilon+\frac{q W}{i K}, 1+2 \Upsilon ;-2 i K r\right)+e^{-i K r} \Phi\left(\Upsilon-\frac{q W}{i K}, 1+2 \Upsilon ; 2 i K r\right), \\
\Phi_{-} & =\frac{1}{i K}\left[e^{i K r} \Phi\left(\Upsilon+\frac{q W}{i K}, 1+2 \Upsilon ;-2 i K r\right)-e^{-i K r} \Phi\left(\Upsilon-\frac{q W}{i K}, 1+2 \Upsilon ; 2 i K r\right)\right],
\end{aligned}
$$

дублет $u_{+}$есть один из используемых ниже дублетов

$$
u_{ \pm}=\left(\begin{array}{c}
1 \\
\frac{\varkappa \pm \Upsilon}{q}
\end{array}\right)
$$

Приведем некоторые частные решения радиальных уравнений (10), которые будут использоваться в дальнейшем.

Одно из решений дается выражением (14) при $A=1, B=0$ и специальном выборе $\Upsilon$ :

$$
U_{(1)}(r ; W)=\left.X(r, \Upsilon, W)\right|_{\Upsilon=\Upsilon_{+}}
$$

где

$$
\Upsilon_{+}= \begin{cases}\sqrt{\varkappa^{2}-q^{2}}, & q \leqslant|\varkappa|, \\ i \sqrt{q^{2}-\varkappa^{2}}, & q>|\varkappa|,\end{cases}
$$


далее положим $\gamma=\sqrt{\varkappa^{2}-q^{2}}, \sigma=\sqrt{q^{2}-\varkappa^{2}}$. Асимптотическое поведение дублета $U_{(1)}(r ; W)$ в начале координат имеет вид

$$
U_{(1)}(r ; W)=(m r)^{\Upsilon_{+}} u_{+}+O\left(r^{\Upsilon_{+}+1}\right), \quad r \rightarrow 0 .
$$

При $\Upsilon_{+} \neq n / 2, n=1,2, \ldots$, мы будем использовать также другое решение

$$
U_{(2)}(r ; W)=\left.X(r, \Upsilon, W)\right|_{\Upsilon=-\Upsilon_{+}}
$$

с асимптотическим поведением

$$
U_{(2)}(r ; W)=(m r)^{-\Upsilon_{+}} u_{-}+O\left(r^{-\Upsilon_{+}+1}\right), \quad r \rightarrow 0 .
$$

При $q \neq q_{\mathrm{c} j}=|\varkappa|=j+1 / 2$, т.е. при $\Upsilon_{+} \neq 0$, решения $U_{(1)}(r ; W)$ и $U_{(2)}(r ; W)$ линейно независимы,

$$
\operatorname{Wr}\left(U_{(1)}, U_{(2)}\right)=-\frac{2 \Upsilon_{+}}{q},
$$

где $\operatorname{Wr}\left(F_{1}, F_{2}\right)=F_{1} i \sigma^{2} F_{2}=f_{1} g_{2}-g_{1} f_{2}$ является вронскианом дублетов $F_{1}=\left(\begin{array}{l}f_{1} \\ g_{1}\end{array}\right)$ и $F_{2}=\left(\begin{array}{c}f_{2} \\ g_{2}\end{array}\right)$.

Из стандартного представления функций $\Phi$ следует, что при действительных $\Upsilon$, $\Upsilon \neq-n / 2$, функции $\Phi_{+}$и $\Phi_{-}$в формулах (15) являются вещественно-целыми функциями от $W$, т.е. они суть целые функции от $W$, принимающие вещественные значения при вещественных $W=E$. Из представлений (16) и (18) следует, что соответствующие дублеты $U_{(1)}(r ; W)$ и $U_{(2)}(r ; W)$ - также вещественно-целые функции от $W$ при действительных $\Upsilon_{+}=\gamma$. Если $\Upsilon_{+}$чисто мнимое, $\Upsilon_{+}=i \sigma$, тогда $U_{(1)}(r ; W)$ и $U_{(2)}(r ; W)$ являются целыми функциями от $W$ и комплексно сопряжены при действительных $W=E, \overline{U_{(1)}(r ; E)}=U_{(2)}(r ; E)$.

Другое полезное решение, нетривиальное при $\Upsilon_{+} \neq n / 2, n=1,2, \ldots$, дается выражением (14) при $A=0$ и специальном выборе $B$ :

$$
\begin{gathered}
V_{(1)}(r ; W)=B(W)(m r)^{\Upsilon_{+}} e^{i K r}\left[\Psi(\alpha, \beta ; z) \vartheta_{+}-b \Psi(\alpha+1, \beta ; z) \vartheta_{-}\right], \\
B(W)=\frac{\Gamma\left(-\Upsilon_{+}+q W / i K\right)}{\Gamma\left(-2 \Upsilon_{+}\right)\left(1-a_{+}\right)} \equiv \frac{1}{\Gamma\left(-2 \Upsilon_{+}\right) \widetilde{B}(W)} .
\end{gathered}
$$

Как и любое решение, $V_{(1)}$ представляется в виде линейной комбинации $U_{(1)}$ и $U_{(2)}$,

$$
V_{(1)}(r ; W)=U_{(1)}(r ; W)+\frac{q}{2 \Upsilon_{+}} \omega(W) U_{(2)}(r ; W),
$$

где

$$
\begin{aligned}
\omega(W) & =-\operatorname{Wr}\left(U_{(1)}, V_{(1)}\right)= \\
& =\frac{2 \Upsilon_{+} \Gamma\left(2 \Upsilon_{+}\right) \Gamma\left(-\Upsilon_{+}+q W / i K\right)\left(1-a_{-}\right)\left(2 e^{-i \pi / 2} K / m\right)^{-2 \Upsilon_{+}}}{q \Gamma\left(-2 \Upsilon_{+}\right) \Gamma\left(\Upsilon_{+}+q W / i K\right)\left(1-a_{+}\right)} \equiv \frac{\widetilde{\omega}(W)}{\Gamma\left(-2 \Upsilon_{+}\right)} .
\end{aligned}
$$

Отметим, что при $\operatorname{Im} W>0$ и $r \rightarrow \infty$ дублет $U_{(1)}(r ; W)$ экспоненциально растет, а дублет $V_{(1)}(r ; W)$ экспоненциально убывает (с полиномиальной точностью).

Дублеты $U_{(2)}$ и $V_{(1)}$ не являются линейно независимыми от $U_{(1)}$ решениями в точках $\Upsilon=\gamma=n / 2$, где $U_{(2)}$ не определен, а $V_{(1)}$ равен нулю. Нам понадобятся их аналоги, определенные в этих точках и обладающие всеми необходимыми свойствами, в частности, вещественно-целые по $W$. K сожалению, мы можем построить 
такие решения только в некоторой окрестности фиксированного $n$. Соответствующим решениям приписывается индекс $n$.

Согласно (21) дублет $V_{(1)}$ стремится к нулю, как $1 / \Gamma(-2 \gamma)$, если $\gamma \rightarrow n / 2$. Тогда из $(22)$ следует, что дублет $U_{(2)}$ имеет сингулярность вида $\Gamma(-2 \gamma)$ в точке $\gamma=n / 2$ и может быть представлен в окрестности этой точки как

$$
U_{(2)}(r ; W)=\Gamma(-2 \gamma) A_{n}(W) U_{(1)}(r ; W)+U_{n(2)}(r ; W),
$$

где

$$
A_{n}(W)=\left.\left(-\frac{2 \gamma}{q} \frac{1}{\widetilde{\omega}(W)}\right)\right|_{\gamma=n / 2},
$$

а $U_{n(2)}(r ; W)$ имеет конечный предел при $\gamma \rightarrow n / 2$ и очевидно удовлетворяет радиальным уравнениям (10). Прямое вычисление ${ }^{14)}$ показывает, что $A_{n}(W)$ является полиномом по $W$ с действительными коэффициентами, и, поскольку $U_{(1)}(r, W)$ и $U_{(2)}(r, W)$ - вещественно-целые функции от $W$, дублет $U_{n(2)}(r ; W)$ также вещественно-целый. Таким образом, мы получаем, что дублет $U_{n(2)}$, определяемый выражением

$$
U_{n(2)}(r ; W)=U_{(2)}(r ; W)-\Gamma(-2 \gamma) A_{n}(W) U_{(1)}(r ; W)
$$

и удовлетворяющий условию

$$
U_{n(2)}(r ; W)=(m r)^{-\gamma} u_{-}+O\left(r^{-\gamma+1}\right), \quad r \rightarrow 0,
$$

является решением радиальных уравнений, которое хорошо определено в некоторой окрестности точки $\gamma=n / 2$ и в само́й этой точке. Это решение линейно не зависит от $U_{(1)}, \operatorname{Wr}\left(U_{(1)}, U_{n(2)}\right)=-2 \gamma / q$, и является вещественно-целым по $W$. Согласно соотношениям (22)-(24) дублет $V_{(1)}$ представляется в окрестности точки $\gamma=n / 2$ в терминах конечных дублетов $U_{(1)}$ и $U_{n(2)}$ следующим образом:

$$
V_{(1)}(r ; W)=\left[1+\frac{q}{2 \gamma} \widetilde{\omega}(W) A_{n}(W)\right] U_{(1)}(r ; W)+\frac{q}{2 \gamma} \omega(W) U_{n(2)}(r ; W),
$$

где согласно $(25)$ и $(23)$ факторы $1+q \widetilde{\omega}(W) A_{n}(W) /(2 \gamma)$ и $\omega(W)$ стремятся к нулю, как $1 / \Gamma(-2 \gamma)$, при $\gamma \rightarrow n / 2$. Это позволяет ввести дублет

$$
V_{n(1)}(r ; W)=\frac{1}{1+q \widetilde{\omega}(W) A_{n}(W) /(2 \gamma)} V_{(1)}(r ; W)=U_{(1)}(r ; W)+\frac{q}{2 \gamma} \omega_{n}(W) U_{n(2)}(r ; W),
$$

где

$$
\omega_{n}(W)=\frac{\omega(W)}{1+q \widetilde{\omega}(W) A_{n}(W) /(2 \gamma)}=\frac{\widetilde{\omega}(W)}{\Gamma(-2 \gamma)\left[1+q \widetilde{\omega}(W) A_{n}(W) /(2 \gamma)\right]},
$$

который, очевидно, является решением радиальных уравнений, хорошо определенным в некоторой окрестности точки $\gamma=n / 2$ и в само́й этой точке, экспоненциально убывающим при $r \rightarrow \infty$. Функция $\omega_{n}(W)$ также хорошо определена в некоторой окрестности точки $\gamma=n / 2$ и в само́й этой точке.

Отметим полезные соотношения

$$
\frac{1}{\omega_{n}(W)} V_{n(1)}(r ; W)=\frac{1}{\omega(W)} V_{(1)}(r ; W),
$$

\footnotetext{
14) $\mathrm{C}$ использованием равенства $\Gamma(w+1)=w \Gamma(w)$.
} 


$$
\frac{1}{\omega_{n}(W)}=\frac{q}{2 \gamma} \Gamma(-2 \gamma) A_{n}(W)+\frac{1}{\omega(W)},
$$

которые имеют строгий смысл при $\gamma \neq n / 2$ и показывают, что правые части непрерывны по $\gamma$ в точках $\gamma=n / 2$.

Дублеты $U_{n(2)}(r ; W)$ и $V_{n(1)}(r ; W)$ представляют собой искомые аналоги дублетов $U_{(2)}(r ; W)$ и $V_{(1)}(r ; W)$, определенные в окрестности точки $\gamma=n / 2$ и в само́й этой точке.

Остается рассмотреть специальный случай $q=q_{\text {с } j}=j+1 / 2$, или $\Upsilon=0$, когда дублеты $U_{(1)}$ и $U_{(2)}$ совпадают, а $V_{(1)}=0$. Пусть $U_{(1)}(r ; W \mid \gamma), U_{(2)}(r ; W \mid \gamma)$ и $V_{(1)}(r ; W \mid \gamma)$ обозначают $U_{(1)}, U_{(2)}$ и $V_{(1)}$ при $\gamma \neq 0$. Дифференцируя радиальные уравнения (10) для $U_{(1)}$ по $\gamma$ при $\gamma=0$, мы можем легко проверить, что дублет

$$
\left.\frac{\partial U_{(1)}(r ; W)}{\partial \gamma}\right|_{\gamma=0}=\lim _{\gamma \rightarrow 0} \frac{U_{(1)}(r ; W \mid \gamma)-U_{(2)}(r ; W \mid \gamma)}{2 \gamma}
$$

является решением этих уравнений при $\gamma=0$. В качестве двух линейно независимых решений радиальных уравнений (10) при $\gamma=0$ мы выберем

$$
\begin{aligned}
& U_{(1)}(r ; W)=U_{(1)}(r ; W \mid 0), \\
& U_{(1)}(r ; W)=u_{+}+O(r), \quad r \rightarrow 0,
\end{aligned}
$$

и

$$
\begin{aligned}
& U_{(2)}^{(0)}(r ; W)=\frac{\partial U_{(1)}(r ; W \mid 0)}{\partial \gamma}-\frac{\zeta}{q_{\mathrm{c} j}} U_{(1)}(r ; W \mid 0), \\
& U_{(2)}^{(0)}(r ; W)=u_{-}^{(0)}(r)+O(r \ln r), \quad r \rightarrow 0,
\end{aligned}
$$

где $u_{+}$и $u_{-}^{(0)}(r)$ имеют вид

$$
u_{+}=\left(\begin{array}{l}
1 \\
\zeta
\end{array}\right), \quad u_{-}^{(0)}(r)=\left(\begin{array}{c}
\ln (m r)-\zeta / q_{\mathrm{c} j} \\
\zeta \ln (m r)
\end{array}\right) .
$$

Вронскиан этих решений задается формулой

$$
\mathrm{Wr}\left(U_{(1)}, U_{(2)}^{(0)}\right)=\frac{1}{q_{\mathrm{c} j}} .
$$

Оба дублета $U_{(1)}$ и $U_{(2)}^{(0)}$ являются вещественно-целыми по $W$.

В качестве аналога $V_{(1)}$ при $\gamma=0$ возьмем дублет

$$
\begin{aligned}
V_{(1)}^{(0)}(r ; W) & =\lim _{\gamma \rightarrow 0}\left[-\Gamma(-2 \gamma) V_{(1)}(r ; W \mid \gamma)\right]= \\
& =-\frac{\Gamma(\alpha)}{1-a} e^{i K r}\left[\Psi(\alpha, 1 ;-2 i K r)+b \Psi(\alpha+1,1 ;-2 i K r) \sigma^{3}\right]\left(\begin{array}{c}
1 \\
i \Lambda
\end{array}\right),
\end{aligned}
$$

где

$$
\alpha=\frac{q_{\mathrm{c} j} W}{i K}, \quad a=\frac{W}{m+i \zeta K}, \quad b=q_{\mathrm{c} j} \frac{\zeta K+i m}{K},
$$


который в терминах $U_{(1)}$ и $U_{(2)}^{(0)}$ задается соотношениями

$$
\begin{aligned}
V_{(1)}^{(0)}(r ; W) & =U_{(2)}^{(0)}(r ; W)+q_{\mathrm{c} j} \omega^{(0)}(W) U_{(1)}(r ; W), \\
\omega^{(0)}(W) & =-\operatorname{Wr}\left(U_{(2)}^{(0)}(r ; W), V_{(1)}^{(0)}(r ; W)\right)= \\
& =\frac{1}{q_{\mathrm{c} j}}\left[\ln \left(\frac{2 e^{-i \pi / 2} K}{m}\right)+\psi\left(-\frac{i q_{\mathrm{c} j} W}{K}\right)+\frac{\zeta(W-m)+i K}{2 q_{\mathrm{c} j} W}-2 \psi(1)\right],
\end{aligned}
$$

где $\psi$ есть символ логарифмической производной Г-функции. Отметим, что при $\operatorname{Im} W>0$ дублет $V_{(1)}^{(0)}(r ; W)$ квадратично интегрируем, $V_{(1)}^{(0)}(r ; W) \in \mathcal{L}^{2}(0, \infty)$, и экспоненциально убывает при $r \rightarrow \infty$.

\section{4. САМОСОПРЯЖЕННЫЕ РАДИАЛЬНЫЕ ГАМИЛЬТОНИАНЫ ДЛЯ РАЗНЫХ ОБЛАСТЕЙ ЗАРЯДА $q$}

4.1. Общая часть. В этом разделе построены с.с. радиальные гамильтонианы $^{15)} \hat{h}$ в гильбертовом пространстве $\mathcal{L}^{2}(0, \infty)$ дублетов как с.с. расширения симметрического радиального оператора $\hat{h}^{(0)}(6)$, ассоциированного с радиальным дифференциальным выражением $\check{h}(7)$, и проанализированы их спектральные свойства.

Процедура расширения включает следующие шаги [22]:

1) вычисление сопряженного оператора $\hat{h}^{*}=\left(\hat{h}^{(0)}\right)^{+}$и оценка его асимметрии в терминах (асимптотических) граничных значений дублетов из области определения $D_{*}$ оператора $\hat{h}^{*}$;

2) построение с.с. расширений $\hat{h}$ оператора $\hat{h}^{(0)}$ как с.с. сужений сопряженного оператора $\hat{h}^{*}$, определяемых некоторыми (асимптотическими) с.с. граничными условиями в начале координат ${ }^{16)}$.

Оказывается ${ }^{17)}$, что результат существенно зависит от значения заряда $q$ : разным областям заряда отвечают различные с.с. радиальные гамильтонианы в том смысле, что они описываются совершенно разными типами (асимптотических) с.с. граничных условий. Более того, для достаточно больших значений заряда с.с. радиальный гамильтониан определен неоднозначно, и при фиксированных $\varkappa$ и $q$ существует однопараметрическое семейство с.с. гамильтонианов. Поэтому наше изложение естественным образом делится на пункты, описывающие определенные области значений заряда; фактически мы имеем четыре области.

Для каждой области мы проведем полный спектральный анализ полученных с.с. гамильтонианов, в частности, найдем их спектры и (обобщенные) собственные функции. Анализ основан на методе направляющих функционалов Крейна и включает следующие этапы:

1) построение направляющего функционала,

2) вычисление резольвенты,

3) вычисление спектральной функции,

${ }^{15)}$ Индексы у величин $\hat{h}, \hat{h}^{(0)}, \check{h}$ опущены, см. замечание в конце раздела 2.

16) Отметим, что этот метод построения $\hat{h}$ позволяет обойтись без вычисления дефектных подпространств и индексов дефекта $\hat{h}^{(0)}$, последние определяются попутно.

17) Фактически это становится ясно уже на первом шаге. 
4) построение так называемых формул обращения, которые являются математически строгими формулами разложения Фурье волновых функций по (обобщенным) собственным функциям с.с. гамильтониана.

Этот строгий анализ сравнивается с эвристическим физическим рассмотрением, основанным, в частности, на правиле "нормировки на $\delta$-функцию" волновых функций непрерывного спектра.

Нашей первой задачей является построение с.с. радиального оператора $\hat{h}$ в соответствии с вышеописанной схемой, что в основном сводится к указанию области определения $D_{h}$ этого оператора, $D_{h^{(0)}} \subset D_{h} \subseteq D_{*}$. Может оказаться, что такая область не единственна, и это действительно реализуется при некоторых значениях числовых параметров, содержащихся в $\check{h}$.

Область определения с.с. дифференциального оператора, заданного на интервале действительной числовой оси, обычно задается так называемыми с.с. граничными условиями на концах интервала для функций из области определения. Наша задача - указать эти условия для дублетов $F \in D_{h}$ на границах $r=0$ и $r=\infty$, которые для дифференциального выражения $\breve{h}(7)$ являются так называемыми сингулярными концами [18]. Сингулярность левого конца $r=0$ связана с неинтегрируемостью свободных членов (коэффициентных функций без производных) в $\breve{h}$. В случае сингулярных концов не существует универсального явного метода формулировки с.с. граничных условий. В нашем случае, когда коэффициентная функция перед производной $d / d r$ не зависит от $r$, а свободные члены ограничены при $r \rightarrow \infty$, проблема с.с. граничных условий связана только с левым концом $r=0$.

Вначале рассмотрим оператор $\hat{h}^{*}$, сопряженный исходному симметрическому оператору $\hat{h}^{(0)}$. Используя известные сведения из теории обобщенных функций (или обобщая известные результаты для скалярных дифференциальных операторов [18] на случай матричных дифференциальных операторов), мы можем легко убедиться, что сопряженный оператор $\hat{h}^{*}$ задается следующим образом:

$$
\hat{h}^{*}:\left\{\begin{array}{l}
D_{h^{*}}=D_{*}=\left\{\begin{array}{l}
F: F \text { абсолютно непрерывны на }(0, \infty), \\
F, \quad \check{h} F=G \in \mathcal{L}^{2}(0, \infty),
\end{array}\right\} \\
\hat{h}^{*} F(r)=\check{h} F(r),
\end{array}\right.
$$

т.е. сопряженный оператор $\hat{h}^{*}$ ассоциирован с тем же самым дифференциальным выражением $\check{h}$, но определен на более широкой области $D_{*}, D_{h^{(0)}} \subset D_{*}$, которая представляет собой так называемую естественную область определения для дифференциального выражения $\breve{h}$. Поскольку коэффициентные функции дифференциального выражения $\breve{h}$ действительны, это означает, что индексы дефекта исходного симметрического оператора $\hat{h}^{(0)}$ равны, так что с.с. расширения $\hat{h}^{(0)}$ существуют для любых значений параметров $\varkappa$ и $q$.

Удобно ввести квадратичную форму асимметрии $\Delta_{*}$ для $\hat{h}^{*}$ соотношением

$$
\begin{aligned}
\Delta_{*}(F) & =\left(F, \hat{h}^{*} F\right)-\left(\hat{h}^{*} F, F\right)=2 i \operatorname{Im}\left(F, \hat{h}^{*} F\right)= \\
& =\int_{0}^{\infty} F^{+}(r)(\check{h} F)(r) d r-\int_{0}^{\infty}(\check{h} F)^{+}(r) F(r) d r .
\end{aligned}
$$


Величина $\Delta_{*}(F)$ является, очевидно, чисто мнимой ${ }^{18)}$. Форма $\Delta_{*}$ есть мера асимметрии оператора $\hat{h}^{*}$, она показывает, в какой мере он отклоняется от симметрического оператора. Если $\Delta_{*} \equiv 0$, оператор $\hat{h}^{*}$ является симметрическим и поэтому c.c. оператором. Это также означает, что $\hat{h}^{(0)}$ - существенно с.с. оператор, и его единственным с.с. расширением является его замыкание, $\hat{h}=\overline{\hat{h}^{(0)}}$, которое совпадает с сопряженным оператором, $\hat{h}=\hat{h}^{*}=(\hat{h})^{+}$. Если $\Delta_{*} \neq 0$, с.с. оператор $\hat{h}=(\hat{h})^{+}$ находится как сужение оператора $\hat{h}^{*}$ на область $D_{h} \subset D_{*}$ такую, что сужение $\Delta_{*}$ на $D_{h}$ равно нулю, и $D_{h}$ является максимальной областью ${ }^{19)}$ [22].

С помощью интегрирования по частям в правой части (37) нетрудно проверить, что $\Delta_{*}$ представляется в виде

$$
\Delta_{*}(F)=[F](\infty)-[F](0),
$$

где

$$
[F](\infty)=\lim _{r \rightarrow \infty}[F](r), \quad[F](0)=\lim _{r \rightarrow 0}[F](r)
$$

суть соответствующие граничные значения квадратичной локальной формы $[F](r)$, определенной соотношением ${ }^{20)}$

$$
[F](r)=-i F^{+}(r) \sigma^{2} F(r)=-[\overline{f(r)} g(r)-\overline{g(r)} f(r)]=-2 i \operatorname{Im} \overline{f(r)} g(r) .
$$

Эти граничные значения существуют в силу существования интегралов в правой части (37). Для краткости мы будем называть их граничными формами на бесконечности и в нуле. Таким образом, форма асимметрии $\Delta_{*}$ определяется граничными формами $^{21)}[F](\infty)$ и $[F](0)$.

Проверим, что для любого $F \in D_{*}$

$$
\lim _{r \rightarrow \infty} F(r)=0 .
$$

Отметим вначале, что из условия $F \in D_{*}$ следует, что $\check{h} F=G$ квадратично интегрируем вместе с $F$. В свою очередь отсюда вытекает, что

$$
\frac{d F(r)}{d r}=\left(-\frac{\varkappa}{r} \sigma^{3}+i \frac{q}{r} \sigma^{2}+m \sigma^{1}\right) F(r)+i \sigma^{2} G(r)
$$

квадратично интегрируема на бесконечности ${ }^{22}$. Теперь остается сослаться на утверждение о том, что если абсолютно непрерывный $F(r)$ квадратично интегрируем на

18) Квадратичная форма асимметрии $\Delta_{*}$ является сужением на диагональ полуторалинейной антиэрмитовой формы асимметрии $\omega_{*}$, определяемой выражением

$$
\omega_{*}\left(F_{1}, F_{2}\right)=\left(F_{1}, \hat{h}^{*} F_{2}\right)-\left(\hat{h}^{*} F_{1}, F_{2}\right)=\int_{0}^{\infty} F_{1}^{+}(r)\left(\breve{h} F_{2}\right)(r) d r-\int_{0}^{\infty}\left(\check{h} F_{1}\right)^{+}(r) F_{2}(r) d r .
$$

Формы $\Delta_{*}$ и $\omega_{*}$ определяют друг друга [22].

19) Т.е. областью, не допускающей дальнейшего расширения с сохранением условия $\Delta_{*} \equiv 0$.

${ }^{20)}$ Квадратичная локальная форма $[F](r)$ является сужением на диагональ полуторалинейной антиэрмитовой локальной формы $\left[F_{1}, F_{2}\right](r)=-i F_{1}^{+}(r) \sigma^{2} F_{2}(r)=-\left[\overline{f_{1}(r)} g_{2}(r)-\overline{g_{1}(r)} f_{2}(r)\right]$. Эти две формы определяют друг друга.

21) Представление (38) есть частный случай так называемого тождества Лагранжа в интегральной форме.

${ }^{22)}$ Дублет $F(r)$ квадратично интегрируем на бесконечности, если $\int_{R}^{\infty} F^{+}(r) F(r) d r<\infty$ для достаточно больших $R$. 
бесконечности вместе со своей производной $d F(r) / d r$, то $F(r) \rightarrow 0$ при $r \rightarrow \infty$, это утверждение является очевидным обобщением хорошо известного аналогичного утверждения для скалярных функций. Таким образом, граничная форма на бесконечности тождественно равна нулю: для любого $F \in D_{*}$

$$
[F](\infty)=0,
$$

и форма асимметрии $\Delta_{*}$ определяется граничной формой в нуле: для любого $F \in D_{*}$

$$
\Delta_{*}(F)=-[F](0)=\left.(\bar{f} g-\bar{g} f)\right|_{r=0} .
$$

Для вычисления этой граничной формы мы должны найти асимптотическое поведение дублетов $F \in D_{*}$ в нуле. Оказывается, что дублеты $F$ могут стремиться к нулю, быть конечными, бесконечными или даже вообще не иметь предела при $r \rightarrow 0$ в зависимости от значений параметров $\varkappa$ и $q$ : при фиксированном $j$ следует различать четыре области заряда $q$, которые определяются двумя характеристическими значениями $q_{\mathrm{u} j}$ и $q_{\mathrm{c} j}\left(q_{\mathrm{u} j}<q_{\mathrm{c} j}\right)$,

$$
\begin{aligned}
& q_{\mathrm{u} j}= \sqrt{\varkappa^{2}-\frac{1}{4}}=\sqrt{j(j+1)} \Longleftrightarrow \Upsilon=\gamma=\frac{1}{2} \Longleftrightarrow \\
& \Longleftrightarrow Z_{\mathrm{u} j}=137 \sqrt{j(j+1)}, \\
& q_{\mathrm{c} j}=|\varkappa|=j+\frac{1}{2} \Longleftrightarrow \Upsilon=0 \Longleftrightarrow Z_{\mathrm{c} j}=137\left(j+\frac{1}{2}\right) .
\end{aligned}
$$

Оценка асимптотического поведения $F \in D_{*}$ в нуле основана на следующем наблюдении. Согласно определению (36) дублеты $F \in D_{*}$ могут рассматриваться как квадратично интегрируемые решения неоднородного дифференциального уравнения

$$
\check{h} F(r)=\left(-i \sigma^{2} \partial_{r}+\frac{\varkappa}{r} \sigma^{1}-\frac{q}{r}+m \sigma^{3}\right) F(r)=G(r)
$$

с правой частью $G$, принадлежащей $\mathcal{L}^{2}(0, \infty)$ и поэтому локально интегрируемой, что позволяет применить к уравнению (40) общую теорию дифференциальных уравнений, см., например, [18]. Для оценки асимптотического поведения $F(r)$ в нуле удобно переписать (40) в виде

$$
\check{h}_{-} F(r)=G_{-}(r)
$$

где

$$
\check{h}_{-}=-i \sigma^{2} \frac{d}{d r}+\frac{\varkappa}{r} \sigma^{1}-\frac{q}{r}, \quad G_{-}(r)=G(r)-m \sigma^{3} F(r) \in \mathcal{L}^{2}(0, \infty) .
$$

Пусть $U_{1}$ и $U_{2}$ - линейно независимые решения однородного дифференциального уравнения $\check{h}_{-} U=0$,

$$
\begin{aligned}
& U_{1}(r)=(m r)^{\Upsilon_{+} u_{+},}, \quad q>0, \\
& U_{2}(r)= \begin{cases}(m r)^{-\Upsilon_{+}} u_{-}, & q>0, \quad q \neq q_{\mathrm{c} j} \quad\left(\Upsilon_{+} \neq 0\right), \\
u_{-}^{(0)}(r), & q=q_{\mathrm{c} j} \quad\left(\Upsilon_{+}=0\right) .\end{cases}
\end{aligned}
$$

Любое решение $F(r)$ неоднородного дифференциального уравнения (41) может быть представлено в виде

$$
F(r)=c_{1} U_{1}(r)+c_{2} U_{2}(r)+I_{1}(r)+I_{2}(r),
$$


где $c_{1}$ и $c_{2}-$ некоторые константы и

$$
\begin{aligned}
& I_{1}(r)=\left\{\begin{array}{l}
\frac{q}{2 \Upsilon_{+}} \int_{r}^{r_{0}}\left[U_{1}(r) \otimes U_{2}(y)\right] G_{-}(y) d y, \quad 0<q \leqslant q_{\mathrm{u} j}, \\
-\frac{q}{2 \Upsilon_{+}} \int_{0}^{r}\left[U_{1}(r) \otimes U_{2}(y)\right] G_{-}(y) d y, \quad q>q_{\mathrm{u} j}, \quad q \neq q_{\mathrm{c} j}, \\
q_{\mathrm{c} j} \int_{0}^{r}\left[U_{1}(r) \otimes U_{2}(y)\right] G_{-}(y) d y, \quad q=q_{\mathrm{c} j},
\end{array}\right. \\
& I_{2}(r)=\left\{\begin{array}{l}
\frac{q}{2 \Upsilon_{+}} \int_{0}^{r}\left[U_{2}(r) \otimes U_{1}(y)\right] G_{-}(y) d y, \quad q>0, \quad q \neq q_{\mathrm{c} j}, \\
-q_{\mathrm{c} j} \int_{0}^{r}\left[U_{2}(r) \otimes U_{1}(y)\right] G_{-}(y) d y, \quad q=q_{\mathrm{c} j},
\end{array}\right.
\end{aligned}
$$

где $\otimes-$ символ тензорного произведения, так что $\left[U_{1}(r) \otimes U_{2}(y)\right]$ есть $(2 \times 2)$-матрица, и $r_{0}>0$ - некоторая константа. Оказывается, что граничная форма $[F](0)$ определяется двумя первыми членами в правой части представления (43) и существенно зависит от значения параметра $\Upsilon$.

4.2. Первая некритическая область. Первая некритическая область значений заряда определяется условием

$$
0<q \leqslant q_{\mathrm{u} j} \Longleftrightarrow \Upsilon_{+}=\gamma \geqslant \frac{1}{2} .
$$

4.2.1. Самосопряженные радиальные гамильтонианы. Представление, заданное формулами (42)-(44), позволяет найти асимптотическое поведение $F \in D_{*}$ в начале координат. Согласно формуле (42) функция $U_{1}(r) \sim r^{\gamma}$ квадратично интегрируема в начале координат, в то время как это свойство не имеет места для функции $U_{2}(r) \sim r^{-\gamma}$. Оценивая интегралы $I_{1}(r)$ и $I_{2}(r)(44)$ с помощью неравенства КошиБуняковского, мы находим, что

$$
I_{1}(r)=O\left(r^{1 / 2}\right), \quad I_{2}(r)=O\left(r^{1 / 2}\right), \quad r \rightarrow 0 .
$$

Отсюда следует, что для принадлежности $F(r)$ к пространству $\mathcal{L}^{2}(0, \infty)$ необходимо, чтобы коэффициент $c_{2}$ перед функцией $U_{2}(r)$ в выражении (43) равнялся нулю, так что

$$
F(r)=c_{1} U_{1}(r)+I_{1}(r)+I_{2}(r)=O\left(r^{1 / 2}\right) \rightarrow 0, \quad r \rightarrow 0,
$$

откуда вытекает, что для любого $F \in D_{*}$

$$
[F](0)=0 .
$$

Это означает, что в области заряда $0<q \leqslant q_{\mathrm{u} j}$, или $\gamma \geqslant 1 / 2$, оператор $\hat{h}=\hat{h}^{*}$ является с.с. оператором и при этом единственным с.с. оператором, ассоциированным с с.с. дифференциальным выражением $\check{h}(7)^{23)}$, так что

$$
\hat{h}:\left\{\begin{array}{l}
D_{h}=\left\{\begin{array}{l}
F: F \text { абсолютно непрерывны на }(0, \infty), \\
F, \check{h} F \in \mathcal{L}^{2}(0, \infty),
\end{array}\right\} \\
\hat{h} F(r)=\check{h} F(r) .
\end{array}\right.
$$

23) Отметим частное следствие: индексы дефекта исходного симметрического оператора $\breve{h}^{(0)}$ в области заряда $0<q \leqslant q_{\mathrm{u}}(\gamma \geqslant 1 / 2)$ равны $(0,0)$. 
Отметим, что этот результат фактически оправдывает стандартное рассмотрение дираковского гамильтониана с $q \leqslant \sqrt{3} / 2$, или $Z \leqslant 118$, в физической литературе, где для $\check{h}$ неявно предполагается естественная область определения ${ }^{24)}$.

Теперь мы приступаем к спектральному анализу полученного с.с. гамильтониана в соответствии со схемой, описанной в пункте 4.1. Необходимая краткая информация о каждом шаге этой схемы приведена в приложении А.

4.2.2. Спектральный анализ. Направляющий функционал. В соответствии с требованиями, изложенными в приложении $\mathrm{A}$, в качестве дублета $U$, определяющего направляющий функционал $\Phi(F ; W)\left(\right.$ А.1), выберем дублет $U_{(1)}(r ; W)$, задаваемый соотношениями (16)-(17),

$$
U(r ; W)=U_{(1)}(r ; W),
$$

дублет $U(r ; W)$ является вещественно-целым, см. раздел 3.

В качестве $\mathcal{D}$ выбираем подпространство дублетов $F(r) \in D_{h}=D_{*}$ с компактным носителем. Очевидно, что $\mathcal{D}$ плотно в $\mathcal{L}^{2}(0, \infty)$.

Направляющий функционал $\Phi$ с такими $U$ и $\mathcal{D}$ является простым, т.е. удовлетворяет свойствам 1-3, приведенным в приложении А. Свойство 1 очевидно, свойство 3 легко проверяется интегрированием по частям, таким образом, следует проверить только выполнение свойства 2: уравнение

$$
\left(\check{h}-E_{0}\right) \Psi(r)=F_{0}(r),
$$

где $F_{0} \in \mathcal{D}$ и удовлетворяет условию

$$
\Phi\left(F_{0} ; E_{0}\right)=\int_{0}^{\infty} U\left(r ; E_{0}\right) F_{0}(r) d r=0,
$$

имеет решение, принадлежащее $\mathcal{D}$.

В этом месте наше изложение разделяется на две части, поскольку удобно рассмотреть случаи $\gamma \neq n / 2$ и $\gamma=n / 2, n=1,2, \ldots$, по отдельности. Сначала рассмотрим случай $\gamma \neq n / 2$, после этого обобщение полученных результатов на случай $\gamma=n / 2$ становится очевидным.

В случае $\gamma \neq n / 2$ любое решение неоднородного уравнения допускает представление

$$
\begin{array}{rl}
\Psi(r)=c_{1} & U\left(r ; E_{0}\right)+c_{2} U_{(2)}\left(r ; E_{0}\right)+\frac{q}{2 \gamma} \int_{r}^{\infty}\left[U\left(r ; E_{0}\right) \otimes U_{(2)}\left(y ; E_{0}\right)\right] F_{0}(y) d y+ \\
+ & \frac{q}{2 \gamma} \int_{0}^{r}\left[U_{(2)}\left(r ; E_{0}\right) \otimes U\left(y ; E_{0}\right)\right] F_{0}(y) d y
\end{array}
$$

где $U_{(2)}\left(r ; E_{0}\right)$ задается формулами $(18)-(20)$. Это представление является копией представления (42)-(44) для решения уравнения (41), где мы положили $r_{0}=\infty$ ввиду компактности носителя дублета $F_{0}$. В этом представлении интегральные слагаемые имеют компактный носитель: если $\operatorname{supp} F_{0} \subset[a, b], 0 \leqslant a<b<\infty$, они равны нулю при $r>b$. Выбирая $c_{1}=c_{2}=0$, мы получаем частное решение $\Psi$ с

${ }^{24)}$ Единственность гамильтониана означает также, что для релятивистской дираковской частицы нельзя ввести понятие $\delta$-потенциала, что может свидетельствовать о неперенормируемости четырехфермионного взаимодействия. 
компактным носителем, имеющее вид

$$
\begin{aligned}
\Psi(r)=\frac{q}{2 \gamma} \int_{r}^{\infty}\left[U\left(r ; E_{0}\right) \otimes U_{(2)}\left(y ; E_{0}\right)\right] F_{0}(y) d y+ \\
+\frac{q}{2 \gamma} \int_{0}^{r}\left[U_{(2)}\left(r ; E_{0}\right) \otimes U\left(y ; E_{0}\right)\right] F_{0}(y) d y .
\end{aligned}
$$

Учитывая асимптотическое поведение дублетов $U=U_{(1)}$ и $U_{(2)}$ в начале координат (см. $(17),(19))$ и оценку (46) для $F_{0}$, мы находим, что асимптотическое поведение этого решения при $r \rightarrow 0$ имеет вид $\Psi(r)=O\left(r^{\delta}\right), \delta=\min (\gamma, 3 / 2)$, откуда следует, что $\Psi \in \mathcal{D}$.

Функция Грина. Найти функцию Грина $G\left(r, r^{\prime} ; W\right), \operatorname{Im} W \neq 0$, с.с. оператора $\hat{h}$, ассоциированного с с.с. дифференциальным выражением $\check{h}$, означает представить единственное решение $\Psi(r) \in D_{h}$ дифференциального уравнения

$$
(\check{h}-W) \Psi(r)=F(r)
$$

с любым $F(r) \in \mathcal{L}^{2}(0, \infty)$ в интегральной форме

$$
\Psi(r)=\int_{0}^{\infty} G\left(r, r^{\prime} ; W\right) F\left(r^{\prime}\right) d r^{\prime} .
$$

Для наших целей достаточно рассмотреть случай $\operatorname{Im} W>0$. Как и любое решение, $\Psi$ допускает представление

$$
\begin{gathered}
\Psi(r)=c_{1} U(r ; W)+c_{2} V(r ; W)+\frac{1}{\omega(W)} \int_{r}^{\infty}[U(r ; W) \otimes V(y ; W)] F(y) d y+ \\
+\frac{1}{\omega(W)} \int_{0}^{r} V(r ; W) \otimes U(y ; W) F(y) d y,
\end{gathered}
$$

где $V(r ; W)=V_{(1)}(r ; W)$ и $\omega(W)$ задаются формулами $(21)-(23)$. Это представление является копией представления $(48)$ с заменой $U_{(2)}$ на $V_{(1)}$. Оно корректно, поскольку $V_{(1)}(r ; W)$ при $\operatorname{Im} W>0$ экспоненциально убывает при $r \rightarrow \infty$. Из условия $\Psi \in \mathcal{L}^{2}(0, \infty)$, достаточного для того, чтобы $\Psi$ принадлежало $D_{h}$ (поскольку тогда автоматически выполняется $\left.\check{h} \Psi=W \Psi+F \in \mathcal{L}^{2}(0, \infty)\right)$, следует, что $c_{1}=c_{2}=0$ : в противном случае $\Psi$ квадратично неинтегрируема на бесконечности (при $c_{1} \neq 0$ ) или в начале координат (при $\left.c_{2} \neq 0\right)$, поскольку $U(r ; W)$ при $\operatorname{Im} W>0$ экспоненциально растет при $r \rightarrow \infty$, а $V(r ; W)$ квадратично неинтегрируема в начале координат. Таким образом, мы получаем, что решение $\Psi \in D_{h}$ уравнения (50) для любого $F \in D_{h}$ представляется в виде

$$
\begin{aligned}
\Psi(r)= & \frac{1}{\omega(W)} \int_{r}^{\infty}[U(r ; W) \otimes V(y ; W)] F(y) d y+ \\
& +\frac{1}{\omega(W)} \int_{0}^{r}[V(r ; W) \otimes U(y ; W)] F(y) d y
\end{aligned}
$$

что является искомым представлением (51) с

$$
G\left(r, r^{\prime} ; W\right)= \begin{cases}\frac{1}{\omega(W)} V(r ; W) \otimes U\left(r^{\prime} ; W\right), & r>r^{\prime}, \\ \frac{1}{\omega(W)} U(r ; W) \otimes V\left(r^{\prime} ; W\right), & r<r^{\prime} .\end{cases}
$$

Это выражение для функции Грина позволяет вычислить спектральную функцию $\sigma(E)$ радиального гамильтониана $\hat{h}$ и записать формулы обращения в соответствии с указаниями приложения А (см. формулы (А.2)-(А.7)). 
Спектральная функция и формулы обращения. Согласно формулам (А.7), (52) и (22) получаем, что

$$
\begin{aligned}
M(c ; W) & =\frac{1}{\omega(W)} U(c ; W) \otimes V(c ; W)= \\
& =\frac{1}{\omega(W)} U(c ; W) \otimes U(c ; W)+\frac{q}{2 \gamma} U(c ; W) \otimes U_{(2)}(c ; W),
\end{aligned}
$$

а тогда, поскольку $U(c ; E)=U_{(1)}(c ; E)$ и $U_{(2)}(c ; E)$ действительны, формулы $($ А.5), (А.6) дают следующее выражение для спектральной функции $\sigma(E)$ радиального гамильтониана $\hat{h}$ :

$$
\frac{d \sigma(E)}{d E}=\frac{1}{\pi} \lim _{\varepsilon \rightarrow 0} \operatorname{Im} \frac{1}{\omega(E+i \varepsilon)},
$$

где $\lim$ в $(53)$, также как и $d \sigma(E) / d E$, понимаются в обобщенном смысле.

Таким образом, спектральная функция определяется (обобщенной) функцией $\operatorname{Im} \omega^{-1}(E)$,

$$
\omega^{-1}(E)=\lim _{\varepsilon \rightarrow 0} \omega^{-1}(E+i \varepsilon) .
$$

В точках, в которых функция

$$
\omega(E)=\lim _{\varepsilon \rightarrow 0} \omega(E+i \varepsilon),
$$

отлична от нуля, $\omega^{-1}(E)=1 / \omega(E)$.

Из явного вида $\omega(W)(23)$ следует, что $\omega(E)$ существует, и ее поведение качественно различается при $|E| \geqslant m$ и $|E|<m$. Поэтому ниже мы отдельно проводим анализ этих двух областей энергий.

Рассмотрим вначале область $|E| \geqslant m$. Прямая проверка показывает, что в этой области энергий функция $\omega(E)$ непрерывна, отлична от нуля и имеет комплексные значения. Следовательно, при $E \geqslant m$ спектральная функция $\sigma(E)$ абсолютно непрерывна и

$$
\begin{gathered}
\frac{d \sigma(E)}{d E}=\frac{1}{\pi} \operatorname{Im} \frac{1}{\omega(E)} \equiv Q^{2}(E), \quad|E| \geqslant m, \\
\omega(E)=\frac{2 \gamma \Gamma(2 \gamma) e^{\epsilon i \pi \gamma} \Gamma(-\gamma+q|E| / i k)[(\varkappa+\gamma) \epsilon k+i q(E-m)](2 k / m)^{-2 \gamma}}{q \Gamma(-2 \gamma) \Gamma(\gamma+q|E| / i k)[(\varkappa-\gamma) \epsilon k+i q(E-m)]}, \\
\epsilon=\frac{E}{|E|}, \quad k=\sqrt{E^{2}-m^{2}} .
\end{gathered}
$$

Теперь рассмотрим случай $|E|<m$. В этой области энергий

$$
\begin{gathered}
\omega(E)=\frac{2 \gamma \Gamma(2 \gamma) \Gamma(-\gamma-q E / \tau)[q(m-E)-(\varkappa+\gamma) \tau](2 \tau / m)^{-2 \gamma}}{q \Gamma(-2 \gamma) \Gamma(\gamma-q E / \tau)[q(m-E)-(\varkappa-\gamma) \tau]}, \\
\tau=\sqrt{m^{2}-E^{2}}
\end{gathered}
$$

$\omega(E)$ действительна, и $\lim _{\varepsilon \rightarrow 0} \omega^{-1}(E+i \varepsilon)$ может быть комплексным только в точках, где $\omega(E)=0$. Поскольку $\Gamma(x)$ не обращается в нуль при действительных $x$, функция $\omega(E)$ может обращаться в нуль только в точках, удовлетворяющих одному из двух условий:

a) $q(m-E)-(\varkappa+\gamma) \tau=0$ или 
б) $\gamma-q E / \tau=-n, n=0,1, \ldots$;

в этих точках $|\Gamma(\gamma-q E / \tau)|=\infty$.

Случай "а" дает $E=-\gamma m / \varkappa$ при $\zeta=1$, однако в этой точке также $-\gamma-q E / \tau=0$, и произведение $\Gamma(-\gamma-q E / \tau)[q(m-E)-(\varkappa+\gamma) \tau] \neq 0$, так что $\omega(E) \neq 0$.

Случай "б" дает $E=E_{n}=m / \sqrt{1+q^{2} /(n+\gamma)^{2}}, n=0,1, \ldots$, однако в точке $E=E_{0}$ при $\zeta=1$ также $q(m-E)-\tau(\varkappa-\gamma)=0$, и поэтому $\mid \Gamma(\gamma-E q / \tau)[q(m-E)-$ $\tau(\varkappa-\gamma)] \mid<\infty$.

Таким образом, $\omega(E)$ обращается в нуль в точках

$$
E=E_{n}=\frac{m}{\sqrt{1+q^{2} /(n+\gamma)^{2}}}, \quad n= \begin{cases}1,2, \ldots, & \zeta=1, \\ 0,1,2, \ldots, & \zeta=-1,\end{cases}
$$

образующих хорошо известный дискретный спектр связанных состояний. Отметим, что дискретный спектр сгущается в точке $E=m$, его асимптотическая форма при $n \rightarrow \infty$ имеет вид

$$
\epsilon_{n} \equiv m-E_{n}=\frac{q^{2}}{2 n^{2}}
$$

и представляет собой хорошо известную нерелятивистскую формулу для энергий связанных состояний.

В окрестностях этих точек

$$
\frac{1}{\omega(E)}=-\frac{Q_{n}^{2}}{E-E_{n}+i \varepsilon}+O(1), \quad Q_{n}^{2}=\lim _{E \rightarrow E_{n}} \frac{E_{n}-E}{\omega(E)} .
$$

Отсюда следует, что при $|E|<m$ спектральная функция $\sigma(E)$ представляет собой ступенчатую функцию со скачками $Q_{n}^{2}$ в точках $E=E_{n}$ (дискретных собственных значениях энергии (56)) и

$$
\frac{d \sigma(E)}{d E}=\sum_{n} Q_{n}^{2} \delta\left(E-E_{n}\right), \quad n=\left\{\begin{array}{ll}
1,2, \ldots, & \zeta=1, \\
0,1,2, \ldots, & \zeta=-1,
\end{array} \quad|E|<m .\right.
$$

Окончательно мы получаем, что спектр $\operatorname{Spec} \hat{h}$ оператора $\hat{h}$ представляет собой объединение дискретного спектра $\bigcup_{n}\left\{E_{n}\right\} \subset(-m, m)$ и непрерывного спектра, содержащего положительную, $[m, \infty)$, и отрицательную, $(-\infty, m]$, части:

$$
\operatorname{Spec} \hat{h}=(-\infty,-m] \cup\left(\bigcup_{n}\left\{E_{n}\right\}\right) \cup[m, \infty) .
$$

Введем обозначения

$$
\begin{gathered}
U_{\text {norm }}(r ; E)=\left\{\begin{array}{l}
Q(E) U(r ; E), \quad|E| \geqslant m, \\
Q_{n} U\left(r ; E_{n}\right), \quad E=E_{n}, \quad|E|<m,
\end{array}\right. \\
\varphi(E)=\left\{\begin{array}{ll}
Q(E) \Phi(E), & |E| \geqslant m, \\
Q_{n} \Phi\left(E_{n}\right), & E=E_{n},
\end{array},|E|<m .\right.
\end{gathered}
$$

Тогда формулы обращения (А.2), (А.3) и равенство Парсеваля (А.4) принимают вид

$$
\varphi(E)=\int_{0}^{\infty} U_{\text {norm }}(r ; E) F(r) d r, \quad E \in(-\infty,-m] \cup\left(\bigcup_{n}\left\{E_{n}\right\}\right) \cup[m, \infty),
$$




$$
\begin{gathered}
F(r)=\int_{-\infty}^{-m} U_{\text {norm }}(r ; E) \varphi(E) d E+\sum_{n} U_{\text {norm }}\left(r ; E_{n}\right) \varphi\left(E_{n}\right)+ \\
\quad+\int_{m}^{\infty} U_{\text {norm }}(r ; E) \varphi(E) d E, \quad n= \begin{cases}1,2, \ldots, & \zeta=1, \\
0,1,2, \ldots, & \zeta=-1,\end{cases} \\
\int_{0}^{\infty}|F(r)|^{2} d r=\int_{-\infty}^{-m}|\varphi(E)|^{2} d E+\sum_{n}\left|\varphi\left(E_{n}\right)\right|^{2}+\int_{m}^{\infty}|\varphi(E)|^{2} d E .
\end{gathered}
$$

Формулы обращения и равенство Парсеваля (61)-(63) традиционно интерпретируются как формулы обобщенного разложения Фурье дублетов $F \in \mathcal{L}^{2}(0, \infty)$ по полному ортонормированному набору собственных функций $U_{\text {norm }}(r ; E)$ с.с. радиального гамильтониана $\hat{h}(47)$, ассоциированного с с.с. дифференциальным выражением $\check{h}(7)$.

Полученные результаты для спектра энергий и (обобщенных) собственных функций совпадают с результатами, которые можно получить стандартным методом, основанным на физических соображениях: собственные функции энергии должны быть локально квадратично интегрируемыми решениями дифференциального уравнения $\breve{h} F=E F$, их модули должны быть ограничены на бесконечности, собственные значения, отвечающие квадратично интегрируемым собственным связанным состояниям, образуют дискретный спектр энергий, и квадратично неинтегрируемые собственные состояния, относящиеся к непрерывному спектру, должны допускать "нормировку на $\delta$-функцию".

В качестве первого примера рассмотрим применение этих соображений к области энергий $|E|<m$. В этой области решения дифференциального уравнения $\breve{h} F=E F$ либо экспоненциально растут, либо экспоненциально убывают (кроме того, они могут быть квадратично неинтегрируемы в начале координат). Так как нужные нам решения должны быть локально квадратично интегрируемы, собственные состояния энергии должны принадлежать $\mathcal{L}^{2}(0, \infty)$. Удобно вначале найти решения, квадратично интегрируемые на бесконечности. Они задаются формулами (21)-(23):

$$
F(r)=c V_{(1)}(r ; E)=c\left[U_{(1)}(r ; E)+\frac{q}{2 \gamma} \omega(E) U_{(2)}(r ; E)\right]
$$

где $c$ - константа. Эти функции квадратично интегрируемы в начале координат (и, следовательно, на всей полуоси) только при выполнении условия $\omega(E)=0$, которое воспроизводит полученные выше результаты, относящиеся к дискретному спектру и соответствующим собственным функциям.

Другим примером применения полученных формул является демонстрация того, что прямым вычислением интегралов можно установить соотношения ортонормальности для собственных функций, которые в физической литературе обычно 
представляются в виде

$$
\begin{aligned}
& \int_{0}^{\infty} U_{\text {norm }}\left(r ; E_{n}\right) U_{\text {norm }}\left(r ; E_{n^{\prime}}\right) d r=\delta_{n n^{\prime}}, \\
& \int_{0}^{\infty} U_{\text {norm }}\left(r ; E_{n}\right) U_{\text {norm }}\left(r ; E^{\prime}\right) d r=0, \\
& \quad \int_{0}^{\infty} U_{\text {norm }}(r ; E) U_{\text {norm }}\left(r ; E^{\prime}\right) d r=\delta\left(E-E^{\prime}\right), \quad|E|,\left|E^{\prime}\right| \geqslant m .
\end{aligned}
$$

Метод вычисления приведен в приложении Б, где он продемонстрирован на примере второй некритической области заряда. K сожалению, прямым вычислением соответствующих интегралов мы не можем установить соотношение полноты для собственных функций, которое обычно представляется в виде

$$
\begin{gathered}
\int_{-\infty}^{-m} U_{\text {norm }}(r ; E) U_{\text {norm }}\left(r^{\prime} ; E\right) d E+\sum_{n} U_{\text {norm }}\left(r ; E_{n}\right) U_{\text {norm }}\left(r^{\prime} ; E_{n}\right)+ \\
+\int_{m}^{\infty} U_{\text {norm }}(r ; E) U_{\text {norm }}\left(r^{\prime} ; E\right) d E=\delta\left(r-r^{\prime}\right),
\end{gathered}
$$

и не знаем никаких эвристических физических аргументов в пользу справедливости этого соотношения.

Теперь остается рассмотреть исключительные случаи $\gamma=n / 2, n=1,2, \ldots$. Как следует из результатов раздела 3 , в окрестности каждой точки $\gamma=n / 2$ и в само́й этой точке мы можем эквивалентно использовать дублеты $U_{n(2)}$ и $V_{n(1)}$ с заменой $\omega(W)$ на $\omega_{n}(W)$ (см. формулы $(26)$ и $\left.(27)\right)$ и получить в точности те же заключения о направляющем функционале и те же результаты для функции Грина, спектральной функции и собственных функций, что и при $\gamma \neq n / 2$. Это очевидным образом вытекает из соотношений (28) и (29), где, в частности, член $q \Gamma(-2 \gamma) A_{n}(W) / 2 \gamma$ в правой части соотношения (29) действителен при действительных $W=E$. Из этих формул также следует, что функция Грина и спектральная функция непрерывны по $\gamma$ в каждой точке $\gamma=n / 2$.

4.3. Вторая некритическая область. Эта область характеризуется условием

$$
q_{\mathrm{u} j}<q<q_{\mathrm{c} j} \Longleftrightarrow 0<\Upsilon_{+}=\gamma<\frac{1}{2} .
$$

4.3.1. Самосопряженные радиальные гамильтонианы. Как и в предыдущем разделе, мы вначале вычислим форму асимметрии $\Delta_{*}(F)(39)$, оценивая асимптотику дублетов $F \in D_{h^{*}}$ в начале координат с помощью представления (42)-(44). В рассматриваемом случае $0<\gamma<1 / 2$ обе функции $U_{(1)}(r) \sim r^{\gamma}$ и $U_{(2)}(r) \sim r^{-\gamma}$ квадратично интегрируемы в начале координат и остаются справедливыми оценки (45), так что для любого $F \in D_{h^{*}}$

$$
F(r)=c_{1}(m r)^{\gamma} u_{+}+c_{2}(m r)^{-\gamma} u_{-}+O\left(r^{1 / 2}\right), \quad r \rightarrow 0, \quad u_{ \pm}=\left(\begin{array}{c}
1 \\
\frac{\varkappa \pm \gamma}{q}
\end{array}\right),
$$

что в свою очередь дает

$$
\Delta_{*}(F)=\frac{2 \gamma}{q}\left(\overline{c_{2}} c_{1}-\overline{c_{1}} c_{2}\right) .
$$

3 Теоретическая и математическая физика, т. 150, № 1, 2007 г. 
Таким образом, форма асимметрии $\Delta_{*}(F)$ оказывается нетривиальной антиэрмитовой квадратичной формой по коэффициентам асимптотики $c_{1}$ и $c_{2}$; это означает, что оператор $\hat{h}^{*}(36)$ не является симметрическим, и возникает проблема построения нетривиальных с.с. расширений исходного симметрического оператора $\hat{h}^{(0)}(6)$.

При решении этой задачи мы будем следовать методу, изложенному в работе [22], который состоит из двух шагов.

1. Приведение квадратичной антиэрмитовой формы $\Delta_{*}$, рассматриваемой как форма по граничным значениям или коэффициентам асимптотики $c_{a}, a=1,2, \ldots$, к канонической диагональной форме с помощью линейного преобразования коэффициентов $c_{a}$ в коэффициенты $c_{+k}, k=1, \ldots, m_{+}$, и $c_{-l}, l=1, \ldots, m_{-}$, так что $\Delta_{*}$ принимает вид

$$
\Delta_{*}=i \kappa\left(\sum_{1}^{m_{+}}\left|c_{+k}\right|^{2}-\sum_{1}^{m_{-}}\left|c_{-l}\right|^{2}\right),
$$

где $\kappa$ - некоторый действительный коэффициент.

2. Наложение связей между $c_{+k}$ и $c_{-l}$ с помощью унитарной $(m \times m)$-матрицы $U$,

$$
c_{-l}=\sum_{1}^{m} U_{k l} c_{+k}, \quad l=1, \ldots, m,
$$

в случае, если индексы инерции формы ${ }^{25)} m_{+}$и $m_{-}$равны, $m_{+}=m_{-}=m$. Каждая такая связь с фиксированной матрицей $U$ обращает в нуль форму $\Delta_{*}$ и задает с.с. (асимптотические) граничные условия, определяющие с.с. расширение исходного симметрического оператора, причем разные матрицы $U$ задают разные с.с. расширения. Обратно, любое с.с. расширение задается некоторой матрицей $U$, так что когда $U$ пробегает группу $U(m)$, реализуется полное $m^{2}$-параметрическое $U(m)$-семейство всех возможных с.с. расширений.

Применим этот метод к рассматриваемому случаю.

С помощью линейного преобразования

$$
c_{1,2} \rightarrow c_{ \pm}=c_{1} \pm i c_{2}
$$

форма асимметрии $\Delta_{*}$ приводится к каноническому диагональному виду:

$$
\Delta_{*}(F)=i \frac{\gamma}{q}\left(\left|c_{+}\right|^{2}-\left|c_{-}\right|^{2}\right) .
$$

Ее индексы инерции равны $(1,1)$, что, в частности, означает, что индексы дефекта оператора $\hat{h}^{(0)}$ при $0<\gamma<1 / 2$ равны $(1,1)$.

Связь

$$
c_{-}=e^{i \theta} c_{+}, \quad 0 \leqslant \theta \leqslant 2 \pi, \quad 0 \sim 2 \pi,
$$

при любом фиксированном $\theta$ задает с.с. граничные условия, определяющие с.с. расширение $\hat{h}_{\theta}$ оператора $\hat{h}^{(0)}$. Разным $\theta$ соответствуют разные с.с. расширения, за исключением эквивалентных случаев $\theta=0$ и $\theta=2 \pi$. Когда $\theta$ пробегает окружность, мы получаем полное однопараметрическое $U(1)$-семейство всех с.с. расширений опеpaтора $\hat{h}^{(0)}$.

\footnotetext{
${ }^{25)}$ Индексы инерции совпадают с индексами дефекта исходного симметрического оператора.
} 
Связь (66) эквивалентна соотношению

$$
c_{2}=\xi c_{1}, \quad-\infty \leqslant \xi=-\operatorname{tg} \frac{\theta}{2} \leqslant+\infty, \quad-\infty \sim+\infty,
$$

значения $\xi= \pm \infty$ эквивалентны, и означают, что $c_{1}=0$; мы будем говорить, что в этих случаях $\xi=\infty$.

Мы переобозначим соответствующий с.с. оператор как $\hat{h}_{\xi}, \hat{h}_{\xi} \equiv \hat{h}_{\theta}$, и пусть $D_{\xi}$ обозначает его область определения. В более подробной форме окончательный результат формулируется следующим образом. Во второй некритической области $0<\gamma<1 / 2$ имеется однопараметрическое $U(1)$-семейство $\left\{\hat{h}_{\xi}\right\}$ с.с. операторов, ассоциированных с с.с. дифференциальным выражением $\breve{h}(7)$. Они определяются с.с. граничными условиями и задаются следующей формулой

$\hat{h}_{\xi}:\left\{\begin{array}{l}D_{\xi}=\left\{\begin{array}{l}F(r): F(r) \text { абсолютно непрерывны на }(0, \infty), \quad F, \check{h} F \in \mathcal{L}^{2}(0, \infty), \\ F(r)=c\left[(m r)^{\gamma} u_{+}+\xi(m r)^{-\gamma} u_{-}\right]+O\left(r^{1 / 2}\right), \quad r \rightarrow 0, \quad-\infty<\xi<+\infty, \\ F(r)=c(m r)^{-\gamma} u_{-}+O\left(r^{1 / 2}\right), \quad r \rightarrow 0, \quad \xi=\infty\end{array}\right\}, \\ \hat{h}_{\xi} F=\check{h} F,\end{array}\right.$

где $c$ - произвольное комплексное число.

Другими словами, в рассматриваемой области заряда $0<\gamma<1 / 2$ одно с.с. дифференциальное выражение $\check{h}$ не определяет однозначно с.с. оператор и необходима дополнительная спецификация области определения в терминах с.с. асимптотических граничных условий, содержащая один действительный параметр $\xi$.

4.3.2. Спектральный анализ. Спектральный анализ в этой области заряда вполне аналогичен анализу, проведенному в пункте 4.2, относящемуся к первой некритической области ${ }^{26)}$. Поэтому мы только отметим необходимые модификации и сформулируем окончательные результаты.

В случае $\xi=0$ соответствующий анализ тождественен анализу пункта 4.2 , и полученные там результаты непосредственно распространяются на область $0<\gamma<$ $1 / 2$ и задаются теми же формулами.

Далее, пока не оговорено противное, предполагается, что $0<|\xi|<\infty, \xi$ произволен, но фиксирован. Случай $\xi=\infty$ будет рассмотрен отдельно ниже.

В качестве дублета $U(r ; W)$, определяющего направляющий функционал (A.1), мы выбираем дублет

$$
U_{\xi}(r ; W)=U_{(1)}(r ; W)+\xi U_{(2)}(r ; W),
$$

удовлетворяющий условию

$$
U_{\xi}(r ; W)=(m r)^{\gamma} u_{+}+\xi(m r)^{-\gamma} u_{-}+O\left(r^{-\gamma+1}\right), \quad r \rightarrow 0,
$$

где $U_{(1)}$ и $U_{(2)}$ определяются формулами $(16)-(20)$; как и ранее, $U_{\xi}(r ; W)$ является вещественно-целым по $W$. Соответствующий направляющий функционал обозначим как $\Phi_{\xi}(F, W)$.

26) Упрощающим обстоятельством является тот факт, что исключены особые случаи $\gamma=0$ и $\gamma=1 / 2$. 
В качестве области $\mathcal{D}$ мы выберем множество $\mathcal{D}_{\xi}$ дублетов с компактным носителем.

Направляющий функционал $\Phi_{\xi}$ с так выбранными $U_{\xi}$ и $\mathcal{D}_{\xi}$ является простым. Действительно, выполнение свойств 1 и 3 очевидно; что касается свойства 2, решение $\Psi$ неоднородного уравнения $\left(\check{h}-E_{0}\right) \Psi=F_{0}, F_{0} \in \mathcal{D}_{\xi}$, при условии $\Psi \in \mathcal{D}_{\xi}$ дается копией формулы (49), где решения $U=U_{(1)}$ и $U_{(2)}$ однородного уравнения заменяются соответственно на решения $U_{\xi}$ и $U_{(1)}$ с вронскианом $\operatorname{Wr}\left(U_{\xi}, U_{(1)}\right)=2 \gamma \xi / q$ :

$$
\begin{aligned}
\Psi(r)=- & \frac{q}{2 \gamma \xi} \int_{r}^{\infty}\left[U_{\xi}\left(r ; E_{0}\right) \otimes U_{(1)}\left(y ; E_{0}\right)\right] F_{0}(y) d y- \\
& \left.\quad-\frac{q}{2 \gamma \xi} \int_{0}^{r} U_{(1)}\left(r ; E_{0}\right) \otimes U_{\xi}\left(y ; E_{0}\right)\right] F_{0}(y) d y .
\end{aligned}
$$

Функция Грина $G_{\xi}\left(r, r^{\prime} ; W\right), \operatorname{Im} W>0$, оператора $\hat{h}_{\xi}$ определяется как ядро интегрального представления любого дублета $\Psi \in D_{\xi}$ в терминах дублета $F=$ $\left(\hat{h}_{\xi}-W\right) \Psi \in \mathcal{L}^{2}(0, \infty):$

$$
\Psi(r)=\int_{0}^{\infty} G_{\xi}\left(r, r^{\prime} ; W\right) F\left(r^{\prime}\right) d r^{\prime} .
$$

Это представление является копией формулы (51). Естественное отличие состоит в замене $U=U_{(1)}$ на $U=U_{\xi}$, поскольку условие $\Psi \in D_{\xi}$ означает, что дублет $\Psi$ удовлетворяет с.с. асимптотическим граничным условиям, заданным в (67). Окончательный результат дается формулой

$$
G\left(r, r^{\prime} ; W\right)= \begin{cases}\frac{1}{\omega_{\xi}(W)} V(r ; W) \otimes U_{\xi}\left(r^{\prime} ; W\right), & r>r^{\prime}, \\ \frac{1}{\omega_{\xi}(W)} U_{\xi}(r ; W) \otimes V\left(r^{\prime} ; W\right), & r<r^{\prime},\end{cases}
$$

где

$$
\begin{aligned}
V(r ; W) & =V_{(1)}(r ; W)=U_{\xi}(r ; W)+\frac{q}{2 \gamma} \omega_{\xi}(W) U_{(2)}(r ; W), \\
\omega_{\xi}(W) & =-\operatorname{Wr}(U, V)=\omega(W)-\frac{2 \gamma \xi}{q},
\end{aligned}
$$

$V_{(1)}(r ; W)$ и $\omega(W)$ задаются формулами $(21)-(23)$, что является копией представления (52) с естественной заменой $U=U_{(1)}$ и $\omega$ на $U=U_{\xi}$ и $\omega_{\xi}$, соответственно.

Отсюда следует, см. (А.5)-(А.7), что

$$
\begin{aligned}
M(c ; W) & =\frac{1}{\omega_{\xi}(W)} U_{\xi}(c ; W) \otimes V(c ; W)= \\
& =\frac{1}{\omega_{\xi}(W)} U_{\xi}(c ; W) \otimes U_{\xi}(c ; W)+\frac{q}{2 \gamma} U_{(2)}(c ; W) \otimes U_{\xi}(c ; W),
\end{aligned}
$$

и, поскольку $U_{\xi}(c ; E)$ и $U_{(2)}(c ; E)$ действительны, что спектральная функция $\sigma_{\xi}(E)$ радиального гамильтониана $\hat{h}_{\xi}, 0<|\xi|<\infty$, определяется выражением

$$
\frac{d \sigma_{\xi}(E)}{d E}=\frac{1}{\pi} \lim _{\varepsilon \rightarrow 0} \operatorname{Im} \frac{1}{\omega_{\xi}(E+i \varepsilon)},
$$

являющимся копией выражения (53) с заменой $\omega$ на $\omega_{\xi}$. Спектральная функция определяется (обобщенной) функцией $\operatorname{Im} \omega_{\xi}^{-1}(E)$,

$$
\omega_{\xi}^{-1}(E)=\lim _{\varepsilon \rightarrow 0} \frac{1}{\omega_{\xi}(E+i \varepsilon)}=\lim _{\varepsilon \rightarrow 0} \frac{1}{\omega(E+i \varepsilon)-2 \gamma \xi / q} .
$$


В точках, в которых функция

$$
\omega_{\xi}(E)=\lim _{\varepsilon \rightarrow 0} \omega_{\xi}(E+i \varepsilon)=\lim _{\varepsilon \rightarrow 0}\left[\omega(E+i \varepsilon)-\frac{2 \gamma \xi}{q}\right]
$$

отлична от нуля, мы имеем $\omega_{\xi}^{-1}(E)=1 / \omega_{\xi}(E)$. Поскольку $\omega_{\xi}(E)$ отличается от $\omega(E)$ на вещественную константу $-2 \gamma \xi / q$, то, как и ранее, естественным образом выделяются две области энергий: $|E| \geqslant m$ и $|E|<m$, и соответствующий анализ в каждой области аналогичен анализу предыдущего пункта.

В области энергий $|E| \geqslant m$ функция $\omega_{\xi}(E)$ непрерывна, отлична от нуля и комплексна, как и $\omega(E)(54)$. Поэтому спектральная функция $\sigma_{\xi}(E)$ при $|E| \geqslant m$ абсолютно непрерывна и

$$
\frac{d \sigma_{\xi}(E)}{d E}=\frac{1}{\pi} \operatorname{Im} \frac{1}{\omega(E)-2 \gamma \xi / q} \equiv Q_{\xi}^{2}(E),
$$

что представляет собой аналог формулы $(54)$ с заменой $\omega(E)$ и $Q(E)$ на $\omega_{\xi}(E)$ и $Q_{\xi}(E)$, соответственно.

В области энергий $|E|<m$ функция $\omega(E)(55)$ действительна, и поэтому функция $\omega_{\xi}(E)$ также действительна. Как и в случае первой некритической области заряда, отсюда следует, что при $|E|<m$ спектральная функция $\sigma_{\xi}(E)$ есть ступенчатая функция со скачками $Q_{\xi, n}^{2}$ в точках $E=E_{\xi, n}$, дискретных собственных значениях энергии, в которых $\omega_{\xi}\left(E_{\xi, n}\right)=0$, и

$$
Q_{\xi, n}^{2}=\lim _{E \rightarrow E_{\xi, n}} \frac{E_{\xi, n}-E}{\omega_{\xi}(E)} .
$$

В результате получаем

$$
\frac{d \sigma_{\xi}(E)}{d E}=\sum_{n} Q_{\xi, n}^{2} \delta\left(E-E_{\xi, n}\right), \quad|E|<m,
$$

т.е. аналог формулы $(57)$ с заменой $E_{n}$ и $Q_{n}$ на $E_{\xi, n}$ и $Q_{\xi, n}$, соответственно.

$\mathrm{K}$ сожалению, найти явную формулу для дискретных собственных значений энергии $E_{\xi, n}$ при $\xi \neq 0$ не удается, мы только отметим, что, как и в первой некритической области заряда, существует бесконечное число таких уровней, сгущающихся в точке $E=m$, и их асимптотическая форма при $n \rightarrow \infty$ дается прежним нерелятивистским выражением, не зависящим от $\xi$,

$$
\epsilon_{\xi, n} \equiv m-E_{\xi, n}=\frac{q^{2}}{2 n^{2}} .
$$

Энергия низшего связанного состояния существенно зависит от $\xi$, и существует значение $\xi$, при котором энергия низшего связанного состояния совпадает с границей $E=-m$ нижнего (позитронного) непрерывного спектра.

Полный спектр Spec $\hat{h}_{\xi}$ радиального гамильтониана $\hat{h}_{\xi}$ дается копией формулы (58) с заменой $E_{n}$ на $E_{\xi, n}$. 
Формулы обращения и равенство Парсеваля,

$$
\begin{aligned}
& \varphi_{\xi}(E)= \int_{0}^{\infty} U_{\xi, \text { norm }}(r ; E) F(r) d r, \quad E \in(-\infty,-m] \cup\left(\bigcup_{n}\left\{E_{\xi, n}\right\}\right) \cup[m, \infty), \\
& F(r)= \int_{-\infty}^{-m} U_{\xi, \text { norm }}(r ; E) \varphi_{\xi}(E) d E+\sum_{n} U_{\xi, \text { norm }}\left(r ; E_{n}\right) \varphi_{\xi}\left(E_{\xi, n}\right)+ \\
& \quad+\int_{m}^{\infty} U_{\xi, \text { norm }}(r ; E) \varphi_{\xi}(E) d E \\
& \int_{0}^{\infty}|F(r)|^{2} d r=\int_{-\infty}^{-m}\left|\varphi_{\xi}(E)\right|^{2} d E+\sum_{n}\left|\varphi_{\xi}\left(E_{\xi, n}\right)\right|^{2}+\int_{m}^{\infty}\left|\varphi_{\xi}(E)\right|^{2} d E,
\end{aligned}
$$

записываются в терминах нормированных собственных функций $U_{\xi, \operatorname{norm}}(r ; E)$ и коэффициентов Фурье $\varphi_{\xi}(E)$, которые определяются копиями формул (59) и (60) с добавлением индекса $\xi$ ко всем символам $Q, U, Q_{n}, E_{n}$ и $\Phi$. Эти соотношения являются копиями формул (61)-(63).

Как и ранее, спектр энергий и (обобщенные) собственные функции радиального гамильтониана $\hat{h}_{\xi}$ могут быть получены стандартным методом, использующим физические соображения.

В качестве примера рассмотрим область энергий $|E|<m$, в которой решения $F$ дифференциального уравнения $\breve{h} F=E F$ экспоненциально растут или экспоненциально убывают при $r \rightarrow \infty$, все решения квадратично интегрируемы в начале координат. Подходящими являются только экспоненциально убывающие решения

$$
\begin{aligned}
F(r) & =c V_{(1)}(r ; E)=c\left[U_{(1)}(r ; E)+\frac{q}{2 \gamma} \omega(E) U_{(2)}(r ; E)\right]= \\
& =c\left[U_{\xi}(r ; E)+\frac{q}{2 \gamma} \omega_{\xi}(E) U_{(2)}(r ; E)\right],
\end{aligned}
$$

где $c$ - константа. Замечательно, что такие решения квадратично интегрируемы на всей полуоси при любых значениях энергии $E \in(-m, m)$. Однако они удовлетворяют с.с. асимптотическим граничным условиям $(67)$, только если $\omega_{\xi}(E)=0$, что воспроизводит результаты, касающиеся собственных значений и собственных функций дискретного спектра оператора $\hat{h}_{\xi}$. Отметим, что с.с. граничные условия имеют физический смысл условия равенства нулю плотности потока вероятности на границе, в данном случае в начале координат. Мы не обращались к этому требованию в первой некритической области заряда, поскольку в этой области оно автоматически удовлетворялось.

Кроме того, мы можем установить соотношения ортонормальности для собственных функций $U_{\xi \text {,norm }}(r ; E)$, которые являются аналогом соотношений $(64)$, прямым вычислением соответствующих интегралов. Эти вычисления, иллюстрирующие общий метод, применимый во всех областях заряда, приведены в приложении Б.

Как и выше, мы не смогли установить соотношения полноты для собственных функций прямым вычислением или на основе эвристических физических соображений. 
Кратко коснемся случая $\xi=\infty$, когда с.с. асимптотические граничные условия для любого $F \in D_{\infty}$ имеют вид

$$
F(r)=c(m r)^{-\gamma} u_{-}+O\left(r^{1 / 2}\right), \quad r \rightarrow 0 .
$$

В качестве дублетов $U$ и $V$ выберем соответственно

$$
\begin{aligned}
U(r ; W) & =U_{\infty}(r ; W)=U_{(2)}(r ; W), \\
U_{\infty}(r ; W) & =(m r)^{-\gamma} u_{-}+O\left(r^{-\gamma+1}\right), \quad r \rightarrow 0,
\end{aligned}
$$

и

$$
\begin{aligned}
& V(r ; W)=\frac{2 \gamma}{q \omega(W)} V_{(1)}(r ; W)=U_{(2)}(r ; W)-\frac{q}{2 \gamma} \omega_{\infty}(W) U_{(1)}(r ; W), \\
& \omega_{\infty}(W)=-\operatorname{Wr}(U, V)=-\frac{4 \gamma^{2}}{q^{2} \omega(W)} .
\end{aligned}
$$

Проводя выкладки, полностью аналогичные случаю $|\xi|<\infty$, находим спектральную функцию $\sigma_{\infty}(E)$,

$$
\frac{d \sigma_{\infty}(E)}{d E}=\frac{1}{\pi} \lim _{\varepsilon \rightarrow 0} \operatorname{Im} \frac{1}{\omega_{\infty}(E+i \varepsilon)}=-\frac{1}{\pi} \frac{q^{2}}{4 \gamma^{2}} \lim _{\varepsilon \rightarrow 0} \operatorname{Im} \omega(E+i \varepsilon) .
$$

Все результаты, касающиеся структуры спектра и формул обращения, остаются полностью аналогичными результатам в случае $|\xi|<\infty$. В частности, спектр связанных состояний определяется полюсами функции $\omega(E)$ в области энергий $|E|<m$; он может быть найден явно.

В заключение отметим, что спектр и нормированные собственные функции $U_{\xi, \text { norm }}(r ; E)$ непрерывны по $\xi$, в том числе в точках $\xi=0$ и $\xi=\infty$.

4.4. Критические заряды. Эта область определяется значениями заряда

$$
q=q_{\mathrm{c} j}=|\varkappa|=j+1 / 2 \Longleftrightarrow \Upsilon_{+}=\gamma=0 .
$$

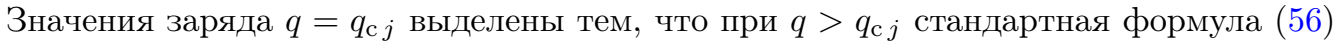
для спектра связанных состояний перестает быть справедливой, давая комплексные значения энергий. Мы увидим, что с математической точки зрения при значениях заряда $q \geqslant q_{\mathrm{c} j}$ с системой не происходит ничего особенного, по крайней мере, по сравнению с предыдущим случаем $q_{\mathrm{u} j}<q<q_{\mathrm{c} j}$.

4.4.1. Самосопряженные радиальные гамильтонианы. При построении с.с. гамильтонианов с критическими зарядами мы будем буквально следовать методу, представленному в предыдущем пункте 4.3, где рассматривалась вторая некритическая область зарядов, и поэтому ограничимся только ключевыми замечаниями.

Из представления (42)-(44) вытекает следующее асимптотическое поведение дублетов $F \in D_{*}$ в случае $q=q_{\mathrm{c} j}$ :

$$
F(r)=c_{1} u_{+}+c_{2} u_{-}^{(0)}(r)+O\left(r^{1 / 2} \ln r\right), \quad r \rightarrow 0,
$$

что для $\Delta_{*}(F)$ дает выражение

$$
\Delta_{*}(F)=\frac{1}{q_{\mathrm{c} j}}\left(\overline{c_{1}} c_{2}-\overline{c_{2}} c_{1}\right),
$$


полностью аналогичное предыдущему, см. формулу (65) с заменами $2 \gamma / q \rightarrow 1 / q_{\mathrm{c}} j$ и $c_{1} \leftrightarrows c_{2}$.

Таким образом, в случае критических зарядов, т.е. при $\gamma=0$, мы также имеем однопараметрическое $U(1)$-семейство $\left\{\hat{h}_{(0) \xi}\right\},-\infty \leqslant \xi \leqslant+\infty$, с.с. операторов, ассоциированных с с.с. дифференциальным выражением $\breve{h}(7)$ и определяемых с.с. асимптотическими граничными условиями,

$\hat{h}_{(0) \xi}:\left\{\begin{array}{l}D_{\xi}^{(0)}=\left\{\begin{array}{l}F(r): F(r) \text { абсолютно непрерывны на }(0, \infty), \quad F, \check{h} F \in \mathcal{L}^{2}(0, \infty), \\ F(r)=c\left(u_{-}^{(0)}(r)+\xi u_{+}\right)+O\left(r^{1 / 2} \ln r\right), \quad r \rightarrow 0,-\infty<\xi<+\infty, \\ F(r)=c u_{+}+O\left(r^{1 / 2} \ln r\right), \quad r \rightarrow 0, \quad \xi=\infty,\end{array}\right. \\ \hat{h}_{(0) \xi} F=\check{h} F,\end{array}\right.$

$D_{\xi}^{(0)}$ - область определения оператора $\hat{h}_{(0) \xi}, \xi=\infty$, отвечает эквивалентным случаям $\xi=+\infty$ и $\xi=-\infty$.

4.4.2. Спектральный анализ. Спектральный анализ следует стандартному пути, представленному в предыдущих пунктах, так что мы приведем только окончательные результаты.

Вначале рассмотрим случай $\xi \neq \infty$. В качестве дублета $U(r ; W)$, определяющего направляющий функционал (А.1), выберем дублет

$$
U_{\xi}^{(0)}(r ; W)=U_{(2)}^{(0)}(r ; W)+\xi U_{(1)}(r ; W),
$$

с асимптотическим поведением

$$
U_{\xi}^{(0)}(r ; W)=u_{-}^{(0)}(r)+\xi u_{+}+O(r \ln r), \quad r \rightarrow 0,
$$

где дублеты $U_{(1)}$ и $U_{(2)}^{(0)}$ задаются формулами $(30)-(33) ; U_{\xi}^{(0)}(r ; W)$ является вещественно-целым по $W$.

В качестве $\mathcal{D}$ выберем множество $\mathcal{D}_{\xi}$ дублетов с компактным носителем.

Направляющий функционал $\Phi_{\xi}^{(0)}$ с такими $U_{\xi}^{(0)}$ и $\mathcal{D}_{\xi}$ простой. В частности, решение $\Psi$ неоднородного уравнения $\left(\check{h}-E_{0}\right) \Psi=F_{0}, F_{0} \in \mathcal{D}_{\xi}$ при условии $\Psi \in \mathcal{D}_{\xi}^{(0)}$ дается копией формулы $(49)$ с заменой $U_{(1)}, U_{(2)}$ и $q /(2 \gamma)=-\left(\operatorname{Wr}\left(U_{(1)}, U_{(2)}\right)^{-1}\right.$ на $U_{\xi}^{(0)}, U_{(1)}$ и $-q_{\mathrm{c} j}=-\left(\operatorname{Wr}\left(U_{\xi}^{(0)}, U_{(1)}\right)^{-1}\right.$, соответственно.

Функция Грина $G_{\xi}^{(0)}\left(r, r^{\prime} ; W\right), \operatorname{Im} W>0$, гамильтониана $\hat{h}_{(0) \xi}$ определяется копией формулы (52) с заменой $U=U_{(1)}, V=V_{(1)}$ и $\omega=-\operatorname{Wr}\left(U_{(1)}, V_{(1)}\right)$ на $U=U_{\xi}^{(0)}$, $V=V_{(1)}^{(0)}$ и $\omega_{\xi}^{(0)}=-\operatorname{Wr}\left(U_{\xi}^{(0)}, V_{(1)}^{(0)}\right)$ соответственно,

$$
G_{\xi}^{(0)}\left(r, r^{\prime} ; W\right)= \begin{cases}\frac{1}{\omega_{\xi}^{(0)}(W)} V_{(1)}^{(0)}(r ; W) \otimes U_{\xi}^{(0)}\left(r^{\prime} ; W\right), & r>r^{\prime}, \\ \frac{1}{\omega_{\xi}^{(0)}(W)} U_{\xi}^{(0)}(r ; W) \otimes V_{(1)}^{(0)}\left(r^{\prime} ; W\right), & r<r^{\prime},\end{cases}
$$

см. формулы $(34),(35) ; V_{(1)}^{(0)}$, удобно представить в виде

$$
V_{(1)}^{(0)}(r ; W)=U_{\xi}^{(0)}(r ; W)+q_{\mathrm{c} j} \omega_{\xi}^{(0)}(W) U_{(1)}(r ; W),
$$


при этом

$$
\omega_{\xi}^{(0)}(W)=-\operatorname{Wr}\left(U_{\xi}^{(0)}, V_{(1)}^{(0)}\right)=\omega^{(0)}(W)-\frac{\xi}{q_{\mathrm{c} j}},
$$

$\omega^{(0)}(W)$ определяется в $(35)$.

Спектральная функция $\sigma_{\xi}^{(0)}(E)$ радиального гамильтониана $\hat{h}_{(0) \xi}$ задается формулой

$$
\frac{d \sigma_{\xi}^{(0)}(E)}{d E}=\frac{1}{\pi} \lim _{\varepsilon \rightarrow 0} \operatorname{Im} \frac{1}{\omega_{\xi}^{(0)}(E+i \varepsilon)}
$$

и определяется (обобщенной) функцией $\operatorname{Im} \omega_{\xi}^{(0)-1}(E)$,

$$
\omega_{\xi}^{(0)-1}(E)=\lim _{\varepsilon \rightarrow 0} \frac{1}{\omega_{\xi}^{(0)}(E+i \varepsilon)}=\lim _{\varepsilon \rightarrow 0} \frac{1}{\omega^{(0)}(E+i \varepsilon)-\xi / q_{\mathrm{c} j}} .
$$

В точках, в которых функция

$$
\omega_{\xi}^{(0)}(E)=\lim _{\varepsilon \rightarrow 0} \omega_{\xi}^{(0)}(E+i \varepsilon)=\omega^{(0)}(E)-\frac{\xi}{q_{\mathrm{c} j}},
$$

где

$$
\omega^{(0)}(E)=\lim _{\varepsilon \rightarrow 0} \omega^{(0)}(E+i \varepsilon)
$$

отлична от нуля, $\omega_{\xi}^{(0)-1}(E)=1 / \omega_{\xi}^{(0)}(E)$.

Как и ранее, естественно выделяются две области значений энергии $|E| \geqslant m$ и $|E|<m$.

В области $|E| \geqslant m$ функция $\omega^{(0)}(E)$ дается выражением

$$
\begin{gathered}
\omega^{(0)}(E)=\frac{1}{q_{\mathrm{c} j}}\left\{\ln \left(2 e^{-i \epsilon \pi / 2} \frac{k}{m}\right)+\psi\left(-i \frac{q_{\mathrm{c} j}|E|}{k}\right)+\frac{i \epsilon k-\zeta m}{2 q_{\mathrm{c} j} E}-2 \psi(1)+\frac{\zeta}{2 q_{\mathrm{c} j}}\right\}, \\
\epsilon=\frac{E}{|E|}, \quad k=\sqrt{E^{2}-m^{2}} .
\end{gathered}
$$

Она непрерывна, отлична от нуля и комплексна, поэтому спектральная функция $\sigma_{\xi}^{(0)}(E)$ при $|E| \geqslant m$ абсолютно непрерывна и

$$
\frac{d \sigma_{\xi}^{(0)}(E)}{d E}=\frac{1}{\pi} \operatorname{Im} \frac{1}{\omega^{(0)}(E)-\xi / q_{\mathrm{c} j}} \equiv\left[Q_{\xi}^{(0)}(E)\right]^{2} .
$$

В области $|E|<m$ функция $\omega^{(0)}(E)$ дается выражением

$$
\begin{gathered}
\omega^{(0)}(E)=\frac{1}{q_{\mathrm{c} j}}\left\{\ln \left(\frac{2 \tau}{m}\right)+\right. \\
\left.\psi\left(-\frac{q_{\mathrm{c} j} E}{\tau}\right)-\frac{\tau+\zeta m}{2 q_{\mathrm{c} j} E}-2 \psi(1)+\frac{\zeta}{2 q_{\mathrm{c} j}}\right\}, \\
\tau=\sqrt{m^{2}-E},
\end{gathered}
$$

она действительна, и поэтому функция $\omega_{\xi}^{(0)}(E)$ также действительна. Как и в предыдущих случаях, спектральная функция $\sigma_{\xi}^{(0)}(E)$ при $|E|<m$ оказывается ступенчатой функцией со скачками

$$
\left(Q_{\xi, n}^{(0)}\right)^{2}=\lim _{E \rightarrow E_{\xi, n}^{(0)}} \frac{E_{\xi, n}^{(0)}-E}{\omega_{\xi}^{(0)}(E)}
$$


в дискретных собственных значениях энергии $E_{\xi, n}^{(0)}$, в которых $\omega_{\xi}^{(0)}\left(E_{\xi, n}^{(0)}\right)=0$, так что

$$
\frac{d \sigma_{\xi}^{(0)}(E)}{d E}=\sum_{n}\left(Q_{\xi, n}^{(0)}\right)^{2} \delta\left(E-E_{\xi, n}^{(0)}\right) .
$$

Как и в предыдущем случае, мы не можем найти явную формулу для $E_{\xi, n}^{(0)}$ (за исключением случая $\xi=\infty$, см. ниже), отметим только, что существует бесконечное число дискретных уровней, которые сгущаются в точке $E=m$, их асимптотическое поведение при $n \rightarrow \infty$ описывается все той же нерелятивистской формулой

$$
\epsilon_{\xi, n}^{(0)} \equiv m-E_{\xi, n}^{(0)}=\frac{q^{2}}{2 n^{2}}
$$

Энергия низшего связанного состояния существенно зависит от $\xi$, и существует значение $\xi$, при котором энергия низшего связанного состояния совпадает с границей $E=-m$ нижнего (позитронного) непрерывного спектра.

Все остальные результаты, касающиеся формул обращения и равенства Парсеваля, записываются в терминах (обобщенных) нормированных собственных функций $U_{\xi, \text { norm }}^{(0)}(r ; E)$ и коэффициентов Фурье $\varphi_{\xi}^{(0)}(E)$ как копии соотношений $(59)-(63)$ с добавлением нижнего индекса $\xi$ и верхнего индекса (0) ко всем символам $Q, U, Q_{n}$, $E_{n}$ и $\Phi$.

Как и ранее, спектр энергий и собственные функции радиального гамильтониана $\hat{h}_{(0) \xi}$ могут быть получены стандартным методом, использующим физические соображения. В качестве примера мы снова рассмотрим область энергий $|E|<m$, в которой решения $F$ дифференциального уравнения $\breve{h} F=E F$ экспоненциально растут или экспоненциально убывают и квадратично интегрируемы в начале координат. Экспоненциально убывающие решения описываются дублетами

$$
F(r)=c V_{(1)}^{(0)}(r ; E)=c\left[U_{\xi}^{(0)}(r ; E)+q_{\mathrm{c} j} \omega_{\xi}^{(0)}(E) U_{(1)}(r ; E)\right],
$$

где $c$ - константа. Они квадратично интегрируемы, $F \in \mathcal{L}^{2}(0, \infty)$, для любых $E$ из интервала $|E|<m$, но удовлетворяют с.с. асимптотическим граничным условиям $(70)$, только если $\omega_{\xi}^{(0)}(E)=0$, что воспроизводит результаты для собственных значений и собственных функций дискретного спектра. С помощью прямого вычисления соответствующих интегралов методом, описанным в приложении Б, можно также проверить, что собственные функции $U_{\xi, \text { norm }}^{(0)}(r ; E)$ удовлетворяют соотношениям ортонормальности, которые представляют собой аналоги соотношений (64).

Кратко коснемся случая $\xi=\infty$, когда с.с. асимптотические граничные условия для любого $F \in D_{\infty}$ имеют вид

$$
F(r)=c u_{+}+O\left(r^{1 / 2} \ln r\right), \quad r \rightarrow 0 .
$$

В качестве дублетов $U$ и $V$ выберем соответственно

$$
\begin{gathered}
U(r ; W)=U_{\infty}^{(0)}(r ; W)=U_{(1)}(r ; W), \\
U_{\infty}^{(0)}(r ; W)=c u_{+}+O(r), \quad r \rightarrow 0,
\end{gathered}
$$

и

$$
V(r ; W)=\frac{1}{q_{\mathrm{c} j} \omega^{(0)}(W)} V_{(1)}^{(0)}(r ; W)=U_{(1)}^{(0)}(r ; W)-q_{\mathrm{c} j} \omega_{\infty}^{(0)}(W) U_{(2)}(r ; W),
$$




$$
\omega_{\infty}^{(0)}(W)=-\operatorname{Wr}\left(U_{(1)}, V\right)=-\frac{1}{q_{\mathrm{c} j}^{2} \omega^{(0)}(W)} .
$$

Совершенно аналогично случаю $|\xi|<\infty$ находим спектральную функцию $\sigma_{\infty}^{(0)}(E)$ :

$$
\frac{d \sigma_{\infty}^{(0)}(E)}{d E}=\frac{1}{\pi} \lim _{\varepsilon \rightarrow 0} \operatorname{Im} \frac{1}{\omega_{\infty}^{(0)}(E+i \varepsilon)}=-\frac{1}{\pi} q_{\mathrm{c} j}^{2} \lim _{\varepsilon \rightarrow 0} \operatorname{Im} \omega^{(0)}(E+i \varepsilon) .
$$

Структура спектра, формулы обращения, соотношения ортонормальности также полностью аналогичны соответствующим результатам в случае $|\xi|<\infty$. В частности, спектр связанных состояний определяется полюсами функции $\omega^{(0)}(E)$ в интервале $|E|<m$. Он может быть вычислен явно и задается формулой (56) с $\gamma=0$, энергия низшего уровня при $\zeta=-1$ равна нулю, $E_{\infty, 0}=0$.

В заключение отметим, что спектр и нормированные собственные функции $U_{\xi, \text { norm }}^{(0)}(r ; E)$ непрерывны по $\xi$, в том числе в точке $\xi=\infty$.

4.5. Сверхкритические заряды. Эта область определяется значениями зарядов

$$
q>q_{\mathrm{c} j}=|\varkappa|=j+1 / 2 \Longleftrightarrow \Upsilon_{+}=i \sigma, \quad \sigma=\sqrt{q^{2}-\varkappa^{2}}>0 .
$$

При построении с.с. гамильтонианов и анализе их спектральных свойств в этой области заряда мы канонически следуем методам, использовавшимся в предыдущих случаях, и поэтому только приведем главные результаты.

4.5.1. Самосопряженные радиальные гамильтонианы. Согласно представлению

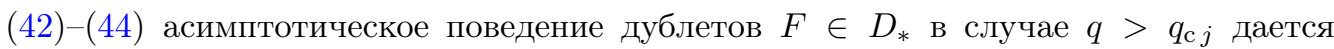
формулой

$$
\begin{gathered}
F(r)=c_{1}(m r)^{i \sigma} u_{+}+c_{2}(m r)^{-i \sigma} u_{-}+O\left(r^{1 / 2}\right), \quad r \rightarrow 0, \\
u_{ \pm}=\left(\begin{array}{c}
1 \\
\frac{\varkappa \pm i \sigma}{q}
\end{array}\right)
\end{gathered}
$$

откуда

$$
\Delta_{*}(F)=\frac{2 i \sigma}{q}\left(\left|c_{1}\right|^{2}-\left|c_{2}\right|^{2}\right)
$$

Таким образом, в случае сверхкритических зарядов, т.е. при $\Upsilon_{+}=i \sigma$, мы имеем однопараметрическое $U(1)$-семейство с.с. операторов $\left\{\hat{h}_{\theta}\right\}, 0 \leqslant \theta \leqslant \pi, 0 \sim \pi$, ассоциированных с дифференциальным выражением $\breve{h}(7)$ и определяемых с.с. асимптотическими граничными условиями ${ }^{27}$,

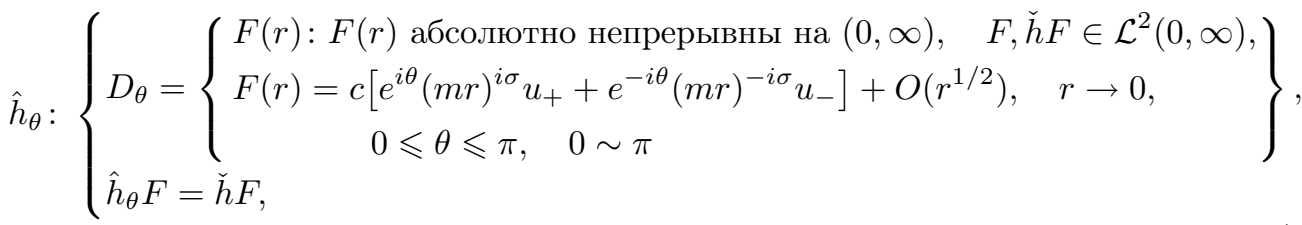

$D_{\theta}$ есть область определения оператора $\hat{h}_{\theta}$.

27) Соотношение $c_{2}=e^{i \theta} c_{1}, 0 \leqslant \theta \leqslant 2 \pi$, задающее с.с. граничные условия (сравни с (66)), эквивалентно соотношению $c_{1}=e^{i \theta} c, c_{2}=e^{-i \theta} c, 0 \leqslant \theta \leqslant \pi$, с заменой $\theta \rightarrow 2 \pi-2 \theta$. 
4.5.2. Спектральный анализ. В качестве дублета $U(r ; W)$, определяющего направляющий функционал (А.1), мы выберем дублет

$$
U_{\theta}(r ; W)=e^{i \theta} U_{(1)}(r ; W)+e^{-i \theta} U_{(2)}(r ; W)
$$

с асимптотическим поведением

$$
U_{\theta}(r ; W)=e^{i \theta}(m r)^{i \sigma} u_{+}+e^{-i \theta}(m r)^{-i \sigma} u_{-}+O(r), \quad r \rightarrow 0,
$$

где $U_{(1)}$ и $U_{(2)}$ определяются формулами (16)-(20) (с $\left.\Upsilon_{+}=i \sigma\right) ; U_{\theta}(r ; W)$ является вещественно-целым по $W$, поскольку $U_{(2)}=\overline{U_{(1)}}$ при $\Upsilon_{+}=i \sigma$ и действительных $W=E$.

В качестве $\mathcal{D}$ мы выберем множество $\mathcal{D}_{\theta}$ дублетов с компактным носителем.

Используя дублеты $U_{\theta}\left(r ; E_{0}\right)$ и $U_{(1)}\left(r ; E_{0}\right)$ для построения решения $\Psi$ неоднородного уравнения $\left(\check{h}-E_{0}\right) \Psi=F_{0}, F_{0} \in \mathcal{D}_{\theta}$ при условии $\Psi_{\theta} \in \mathcal{D}_{\theta}$, мы проверяем, что направляющий функционал $\Phi_{\theta}$ простой.

Функция Грина $G_{\theta}\left(r, r^{\prime} ; W\right), \operatorname{Im} W>0$, гамильтониана $\hat{h}_{\theta}$ строится в терминах дублетов $U=U_{\theta}$ и $V=V_{\theta}$, где

$$
\begin{gathered}
V_{\theta}(r ; W)=\frac{2}{e^{-i \theta}+e^{i \theta} \widetilde{\omega}(W)} V_{(1)}(r ; W)=U_{\theta}(r ; W)-\frac{q}{4 \sigma} \omega_{\theta}(W) \widetilde{U}_{\theta}(r ; W), \\
\widetilde{U}_{\theta}(r ; W)=\frac{1}{i}\left[e^{i \theta} U_{(1)}(r ; W)-e^{-i \theta} U_{(2)}(r ; W)\right], \\
\widetilde{\omega}(W)=\frac{q}{2 i \sigma} \omega(W), \quad \omega_{\theta}(W)=-\mathrm{Wr}\left(U_{\theta}, V_{\theta}\right)=-\frac{4 i \sigma}{q} \frac{1-\widetilde{\omega}(W) e^{2 i \theta}}{1+\widetilde{\omega}(W) e^{2 i \theta}},
\end{gathered}
$$

$V_{(1)}(r ; W)$ и $\omega(W)$ заданы в $(21)-(23)\left(\right.$ с $\left.\Upsilon_{+}=i \sigma\right) ; \widetilde{U}_{\theta}(r ; W)$ является вещественноцелым по $W$. В результате получаем

$$
G_{\theta}\left(r, r^{\prime} ; W\right)= \begin{cases}\frac{1}{\omega_{\theta}(W)} V_{\theta}(r ; W) \otimes U_{\theta}\left(r^{\prime} ; W\right), & r>r^{\prime}, \\ \frac{1}{\omega_{\theta}(W)} U_{\theta}(r ; W) \otimes V_{\theta}\left(r^{\prime} ; W\right), & r<r^{\prime} .\end{cases}
$$

Спектральная функция $\sigma_{\theta}(E)$ радиального гамильтониана $\hat{h}_{\theta}$ задается формулой

$$
\frac{d \sigma_{\theta}(E)}{d E}=\frac{1}{\pi} \lim _{\varepsilon \rightarrow 0} \operatorname{Im} \frac{1}{\omega_{\theta}(E+i \varepsilon)}
$$

и определяется (обобщенной) функцией $\operatorname{Im} \omega^{-1}(E)$,

$$
\omega_{\theta}^{-1}(E)=\lim _{\varepsilon \rightarrow 0} \frac{1}{\omega_{\theta}(E+i \varepsilon)} .
$$

В точках, в которых функция

$$
\omega_{\theta}(E)=\lim _{\varepsilon \rightarrow 0} \omega_{\theta}(E+i \varepsilon)
$$

отлична от нуля, $\omega_{\theta}^{-1}(E)=1 / \omega_{\theta}(E)$.

Как и ранее, естественно выделяются две области значений энергии: $|E| \geqslant m$ и $|E|<m$.

В области $|E| \geqslant m$ функция $\omega_{\theta}(E)$ непрерывна, отлична от нуля и комплексна, поэтому спектральная функция $\sigma_{\theta}(E)$ при $|E| \geqslant m$ абсолютно непрерывна и

$$
\frac{d \sigma_{\theta}(E)}{d E}=\frac{1}{\pi} \operatorname{Im} \frac{1}{\omega_{\theta}(E)} \equiv Q_{\theta}^{2}(E) .
$$


В области $|E|<m$

$$
\widetilde{\omega}(E)=\frac{\Gamma(2 i \sigma)}{\Gamma(-2 i \sigma)} \frac{\Gamma(-i \sigma-E q / \tau)}{\Gamma(i \sigma-E q / \tau)} \frac{\tau(\varkappa+i \sigma)-q(m-E)}{\tau(\varkappa-i \sigma)-q(m-E)}(2 \tau / m)^{-2 i \sigma} \equiv e^{-2 i \Theta(E)},
$$

поэтому функция

$$
\omega_{\theta}(E)=\frac{4 \sigma}{q} \operatorname{tg}(\Theta(E)-\theta)
$$

действительна.

Таким образом, спектральная функция $\sigma_{\theta}(E)$ при $|E|<m$ является ступенчатой функцией со скачками

$$
Q_{\theta, n}^{2}=\lim _{E \rightarrow E_{\theta, n}} \frac{E_{\theta, n}-E}{\omega_{\theta}(E)},
$$

локализованными в дискретных точках $E_{\theta, n}$, в которых $\omega_{\theta}\left(E_{\theta, n}\right)=0$, так что

$$
\frac{d \sigma_{\theta}(E)}{d E}=\sum_{n} Q_{\theta, n}^{2} \delta\left(E-E_{\theta, n}\right) .
$$

Явную формулу для дискретных собственных значений $E_{\theta, n}$ найти не удается. Мы только отметим, что имеется бесконечное число дискретных уровней, которые сгущаются в точке $E=m$. Их асимптотический вид при $n \rightarrow \infty$ задается прежней нерелятивистской формулой

$$
\epsilon_{\theta, n} \equiv m-E_{\theta, n}=\frac{q^{2}}{2 n^{2}} .
$$

Энергия низшего связанного состояния существенно зависит от $\theta$, и существует значение $\theta$, при котором энергия низшего связанного состояния совпадает с границей $E=-m$ нижнего (позитронного) непрерывного спектра.

Формулы обращения и равенство Парсеваля, записанные в терминах нормированных (обобщенных) собственных функций $U_{\theta, \text { norm }}(r ; E)$ и коэффициентов Фурье $\varphi_{\theta}(E)$, представляют собой копии формул (59)-(63).

Как и в предыдущих пунктах, справедливо замечание о применимости стандартного метода вычисления спектра энергий и собственных функций, использующего физические соображения. В качестве примера мы рассмотрим область энергий $|E|<m$, в которой решения $F$ дифференциального уравнения $\breve{h} F=E F$ экспоненциально растут или экспоненциально убывают при $r \rightarrow \infty$, любое решение квадратично интегрируемо в начале координат. Подходящими являются только экспоненциально убывающие решения,

$$
F=c V_{\theta}(r ; E)=c\left[U_{\theta}(r ; E)-\frac{q}{4 \sigma} \omega_{\theta}(E) \widetilde{U}_{\theta}(r ; E)\right],
$$

где $c$ - константа. Они квадратично интегрируемы, $F \in \mathcal{L}^{2}(0, \infty)$, при любых $E$ из интервала $|E|<m$, но удовлетворяют с.с. граничным условиям (72) только при $\omega_{\theta}(E)=0$, что воспроизводит результаты для собственных значений энергии и собственных функций дискретного спектра. С помощью прямого вычисления соответствующих интегралов методом, описанным в приложении Б, можно также проверить, что собственные функции $U_{\theta, \text { norm }}(r ; E)$ удовлетворяют соотношениям ортонормальности, которые представляют собой аналоги соотношений (64). 
В заключение укажем, что число с.с. расширений полного гамильтониана Дирака (точнее, число независимых параметров с.с. расширений) зависит от значений заряда $q$ (от значения $Z$ ) следующим образом: легко убедиться, что в интервале $q_{n}<q \leqslant q_{n+1}$, где

$$
q_{n}= \begin{cases}0, & n=0, \\ \sqrt{n^{2}-1 / 4}, & n=1,2, \ldots,\end{cases}
$$

число независимых параметров с.с. расширений равно $2 n$. Это следует из того, что полный гамильтониан Дирака есть прямая сумма своих частей, унитарно эквивалентных радиальным гамильтонианам, см. (9), (8).

ПРИЛОЖЕНИЕ А

Один из методов нахождения спектра с.с. дифференциального оператора и его полной системы (обобщенных) собственных функций и построения соответствующего разложения Фурье по этим собственным функциям (так называемых формул обращения) основан на методе Крейна направляющих функционалов. Для обыкновенных скалярных дифференциальных операторов этот метод описан в [18], однако он непосредственно обобщается на случай обыкновенных матричных операторов. Мы приведем здесь ключевые моменты этого метода в приложении к нашему случаю, когда достаточно использовать только один направляющий функционал. Это означает, что спектр оператора простой ${ }^{28)}$; мы будем называть такой функционал простым направляющим функционалом.

По определению направляющий функционал $\Phi(F ; W)$ для с.с. оператора $\hat{h}$, ассоциированного с дифференциальным выражением $\check{h}$, есть функционал вида

$$
\Phi(F ; W)=\int_{0}^{\infty} U(r ; W) F(r) d r,
$$

где $U(r ; W)$ - вещественно-целое по $W$ решение однородного уравнения

$$
(\check{h}-W) U=0,
$$

и $F(r)$ принадлежит некоторому подпространству $\mathcal{D} \subset D \cap D_{h}$, плотному в $\mathcal{L}^{2}(0, \infty)$, где $D$ - пространство функций с компактным носителем. Направляющий функционал $\Phi(F ; W)$ называется простым, если он удовлетворяет следующим условиям:

$1)$ при фиксированном $F$ функционал $\Phi(F ; W)$ является целой функцией от $W$;

2) если

$$
\Phi\left(F_{0} ; E_{0}\right)=0, \quad \operatorname{Im} E_{0}=0, \quad F_{0} \in \mathcal{D},
$$

то неоднородное уравнение

$$
\left(\check{h}-E_{0}\right) \Psi=F_{0}
$$

имеет решение $\Psi \in \mathcal{D}$;

3) $\Phi(\hat{h} F ; W)=W \Phi(F ; W)$.

Отметим, что существование простого направляющего функционала обуславливается существованием подходящих $U$ и $\mathcal{D}$. Руководящий принцип для выбора $U(r ; W)$ состоит в том, что его поведение при $r \rightarrow 0$ должно отвечать асимптотике,

\footnotetext{
28) Определение см. в [18]. В физической терминологии это означает, что спектр невырожден.
} 
допустимой для дублетов из $D_{h}$, или, нестрого говоря, $U(r ; W)$ в начале координат должен принадлежать $D_{h}$. Это соответствует обычному физическому требованию, чтобы (обобщенные) собственные функции оператора $\hat{h}$, вообще говоря, неинтегрируемые квадратично, но "нормируемые на $\delta$-функцию", удовлетворяли с.с. граничным условиям, которые определяют $\hat{h}$.

Если простой направляющий функционал существует, то с.с. оператор $\hat{h}$ обладает следующими спектральными свойствами:

1) спектр $\hat{h}$ простой, и существует спектральная функция $\sigma(E)$, т.е. действительная неубывающая функция, непрерывная справа и такая, что множество точек спектра совпадает с множеством точек роста ${ }^{29)}$ функции $\sigma(E)$;

2) справедливы формулы обращения

$$
\begin{aligned}
\Phi(E) & =\int_{0}^{\infty} U(r ; E) F(r) d r \\
F(r) & =\int_{-\infty}^{\infty} \Phi(E) U(r ; E) d \sigma(E)
\end{aligned}
$$

и равенство Парсеваля

$$
\int_{0}^{\infty}|F(r)|^{2} d r=\int_{-\infty}^{\infty}|\Phi(E)|^{2} d \sigma(E),
$$

где $\Phi(E) \in L_{\sigma}^{2}(-\infty, \infty), F(r) \in \mathcal{L}^{2}(0, \infty)$, функция $U(r ; E)$ в подынтегральных выражениях в формулах (А.2) и (А.3) может быть доопределена нулем вне точек спектра оператора $\hat{h}$ (вне точек роста $\sigma(E)$ ), и сходимость интегралов в формулах (А.2) и (А.3), вообще говоря, должна пониматься в смысле сходимости по метрике соответствующих пространств $L_{\sigma}^{2}(-\infty, \infty)$ и $\mathcal{L}^{2}(0, \infty)$. Это означает, что набор (обобщенных) собственных функций $\{U(r ; E), E \in \operatorname{Spec} \hat{h}\}$ оператора $\hat{h}$ образует полную ортогональную систему.

Спектральная функция $\sigma(E)$ может быть выражена через резольвенту оператоpa $\hat{h}$. Как известно, см. [18], резольвента $\widehat{R}(W)=(\hat{h}-W)^{-1}$ при $\operatorname{Im} W \neq 0$ является интегральным оператором с ядром $G\left(r, r^{\prime} ; W\right)$ (функцией Грина). Спектральная функция $\sigma(E)$ следующим образом выражается через функцию Грина:

$$
\begin{gathered}
U(c ; E) \otimes U(c ; E) d \sigma(E)=d \mathcal{M}(c ; E), \\
\mathcal{M}(c ; E)=\lim _{\delta \rightarrow+0} \lim _{\varepsilon \rightarrow+0} \frac{1}{\pi} \int_{\delta}^{E+\delta} \operatorname{Im} M\left(c ; E^{\prime}+i \varepsilon\right) d E^{\prime}, \\
M(c ; W)=G(c-0, c+0 ; W),
\end{gathered}
$$

где $c$ - произвольная внутренняя точка интервала $(0, \infty)$. Отметим, что при любом $E$ один из диагональных элементов матрицы $U(c ; E) \otimes U(c ; E)$ отличен от нуля. Конечно, $\sigma(E)$ не зависит от $c$.

${ }^{29)}$ Множество точек роста функции $\sigma(E)$ является дополнением к открытому множеству точек постоянства функции $\sigma(E)$. Точка $E_{0}$ является точкой постоянства функции $\sigma(E)$, если существует окрестность точки $E_{0}$, в которой $\sigma(E)$ постоянна. 
ПРИЛОЖКНИЕ Б

В этом приложении мы описываем метод вычисления так называемых интегралов перекрытия для решений дифференциального уравнения $\breve{h} F=E F$ с разными $E$. Он основан на интегральном тождестве Лагранжа и на оценке асимптотического поведения решений на границах, т.е. в начале координат и на бесконечности. Мы применим этот метод к доказательству ортонормальности (обобщенных) собственных функций радиального гамильтониана на примере второй некритической области заряда. Для двух дублетов $F$ и $F^{\prime}$ мы называем интеграл ${ }^{30)}$

$$
\int_{0}^{\infty} F(r ; W) F^{\prime}\left(r ; W^{\prime}\right) d r=\lim _{R \rightarrow \infty, \epsilon \rightarrow 0} \int_{\epsilon}^{R} F(r ; W) F^{\prime}\left(r ; W^{\prime}\right) d r
$$

интегралом перекрытия для этих дублетов. Пусть $F$ и $F^{\prime}$ удовлетворяют соответствующим однородным уравнениям

$$
(\check{h}-W) F(r ; W)=0, \quad\left(\check{h}-W^{\prime}\right) F^{\prime}\left(r ; W^{\prime}\right)=0 .
$$

Тогда для интеграла перекрытия справедливо следущее равенство:

$$
\int_{0}^{\infty} F(r ; W) F^{\prime}\left(r ; W^{\prime}\right) d r=I^{\infty}-I_{0},
$$

где

$$
\begin{aligned}
I^{\infty} & =\lim _{r \rightarrow \infty} \frac{\operatorname{Wr}\left(r ; F, F^{\prime}\right)}{W-W^{\prime}}, \\
I_{0} & =\lim _{r \rightarrow 0} \frac{\operatorname{Wr}\left(r ; F, F^{\prime}\right)}{W-W^{\prime}} .
\end{aligned}
$$

Равенство (Б.1) является специальным случаем интегрального тождества Лагранжа. В дальнейшем нас интересует случай действительных $W$ и $W^{\prime}, W=E$ и $W^{\prime}=E^{\prime}$. Интеграл перекрытия понимается как обобщенная функция от $E$ и $E^{\prime}$. Вычисление интегралов перекрытия сводится таким образом к вычислению асимптотик вронскиана соответствующих дублетов на границах.

Начнем с вычисления асимптотик некоторых базисных функций и дублетов.

Пусть $|E| \geqslant m$, в этом случае имеем $K=|E| k / E, k=\sqrt{E^{2}-m^{2}}$. Пусть $r \rightarrow \infty$. Используя известные асимптотики функций $\Phi(\alpha, \beta ; x)$ (см., например, [21]), имеем

$$
\begin{gathered}
\Phi\left(\alpha, \beta ; 2 i \xi_{1} k r\right) \rightarrow \frac{\Gamma(1+2 \Upsilon)}{\Gamma\left(1+\Upsilon-i \xi_{2} q E / k\right)} e^{i \xi_{1} \pi \Upsilon / 2} e^{-\xi_{1} \xi_{2} \pi E q /(2 k)}(2 k r)^{-\Upsilon-i \xi_{2} E q / k}, \\
\alpha=\Upsilon+i \frac{\xi_{2} q E}{k}, \quad \xi_{1}= \pm 1, \quad \xi_{2}= \pm 1, \quad \beta=1+2 \Upsilon
\end{gathered}
$$

где $\Upsilon$ - любое действительное или чисто мнимое число, $\Upsilon \neq-n / 2, n=1,2, \ldots$. Если $\Upsilon$ действительно и $\Upsilon \neq-n / 2, n=1,2, \ldots$, то

$$
\begin{aligned}
& (m r)^{\Upsilon} \Phi_{+}(r, \Upsilon, E, k) \rightarrow 2 \Delta(\Upsilon, E) \cos \psi(r ; \Upsilon, E), \\
& (m r)^{\Upsilon} \Phi_{-}(r, \Upsilon, E, k) \rightarrow \frac{2}{k} \Delta(\Upsilon, E) \sin \psi(r ; \Upsilon, E),
\end{aligned}
$$

${ }^{30)} \mathrm{B}$ этом приложении обозначение типа $F^{\prime}$ означает другую функцию, а не производную. 
где

$$
\begin{aligned}
\Delta(\Upsilon, E) & =\frac{\Gamma(1+2 \Upsilon)(2 k / m)^{-\Upsilon} e^{-\pi E q /(2 k)}}{|\Gamma(1+\Upsilon+i q E / k)|}, \\
\psi(r ; \Upsilon, E) & =k r+\frac{q E}{k} \ln (2 k r)-\frac{\pi \Upsilon}{2}-\psi_{\Gamma}(\Upsilon, E), \\
\psi_{\Gamma}(\Upsilon, E) & =\arg \Gamma(1+\Upsilon+i q E / k),
\end{aligned}
$$

так что

$$
X(r, \Upsilon, E, k) \rightarrow \Delta(\Upsilon, E)\left[\cos \psi(r ; \Upsilon, E) u_{+}+\frac{\sin \psi(r ; \Upsilon, E)}{k}\left(\begin{array}{c}
\frac{(m+W)(\varkappa+\gamma)}{q} \\
m-E
\end{array}\right)\right]
$$

Пусть $|E|<m$. Для наших целей достаточно знать, что дублеты $U\left(r ; E_{n}\right)$ и $V(r ; E)$ экспоненциально убывают при $r \rightarrow \infty$.

Мы будем использовать соотношение

$$
\begin{aligned}
\frac{E}{|E|} \frac{\sin \left[\psi(r ; \Upsilon, E) \pm \psi\left(r ; \Upsilon^{\prime} E^{\prime}\right)\right]}{E-E^{\prime}} & \rightarrow \frac{|E| \frac{\sin \left[\left(k-k^{\prime}\right) r\right]}{k}}{k-k^{\prime}} \rightarrow \\
& \rightarrow \frac{|E|}{k} \pi \delta\left(k-k^{\prime}\right)=\pi \delta\left(E-E^{\prime}\right), \quad r \rightarrow \infty,
\end{aligned}
$$

справедливое в обобщенном смысле. Выражения вида

$$
\begin{aligned}
& a_{ \pm}\left(E, E^{\prime}\right) \sin \left[\psi(r ; \Upsilon, E) \pm \psi\left(r ; \Upsilon^{\prime}, E^{\prime}\right)\right], \\
& b_{ \pm}\left(E, E^{\prime}\right) \cos \left[\psi(r ; \Upsilon, E) \pm \psi\left(r ; \Upsilon^{\prime}, E^{\prime}\right)\right],
\end{aligned}
$$

где $a_{ \pm}\left(E, E^{\prime}\right), b_{ \pm}\left(E, E^{\prime}\right)$ конечны при $E=E^{\prime}$, будут называться быстро осциллирующими (БО); такие выражения имеют нулевой предел в обобщенном смысле при $r \rightarrow \infty$.

Это позволяет найти пределы $I^{\infty}$ для базисных дублетов. Имеем

$$
\begin{gathered}
U_{(1)}=U_{(1)}(r ; \gamma, E), \quad U_{(1)}^{\prime}=U_{(1)}\left(r ; \gamma, E^{\prime}\right), \quad \gamma>0, \\
\left(E-E^{\prime}\right)^{-1} W\left(r ; U_{(1)}, U_{(1)}^{\prime}\right) \rightarrow \\
\rightarrow A(\gamma, E) \frac{E}{|E|} \frac{\sin \left[\psi(r ; \gamma, E)-\psi\left(r ; \gamma, E^{\prime}\right)\right]}{\pi\left(E-E^{\prime}\right)}+\mathrm{BO} \rightarrow \\
\rightarrow A(\gamma, E) \delta\left(E-E^{\prime}\right), \quad r \rightarrow \infty ; \\
\left(E-E^{\prime}\right)^{-1} W\left(r ; U_{(1)}, U_{(2)}^{\prime}\right) \rightarrow \quad 0<\gamma<1 / 2, \\
\rightarrow B(\gamma, E) \frac{E}{|E|} \frac{\sin \left[\psi(r ; \gamma, E)-\psi\left(r ;-\gamma, E^{\prime}\right)\right]}{\pi\left(E-E^{\prime}\right)}-U_{(2)}\left(r ; \gamma, E^{\prime}\right), \quad \text { (Б.5) } \\
-B(\gamma, E) \frac{\gamma k}{q|E|} \frac{\cos \left[\psi(r ; \gamma, E)-\psi\left(r ;-\gamma, E^{\prime}\right)\right]}{\pi\left(E-E^{\prime}\right)}+\mathrm{BO} \rightarrow \\
\rightarrow B(\gamma, E) \delta\left(E-E^{\prime}\right)-B(\gamma, E) \frac{\gamma k}{q|E|} \frac{\cos \left[\psi(r ; \gamma, E)-\psi\left(r ;-\gamma, E^{\prime}\right)\right]}{\pi\left(E-E^{\prime}\right)}, \quad r \rightarrow \infty \\
U_{(2)}=U_{(2)}(r ; \gamma, E), \quad U_{(1)}^{\prime}=U_{(1)}\left(r ; \gamma, E^{\prime}\right), \quad 0<\gamma<1 / 2,
\end{gathered}
$$




$$
\begin{gathered}
\left(E-E^{\prime}\right)^{-1} W\left(r ; U_{(2)}, U_{(1)}^{\prime}\right) \rightarrow \\
\rightarrow B(\gamma, E) \delta\left(E^{\prime}-E\right)-B(\gamma, E) \frac{\gamma k}{q|E|} \frac{\cos \left[\psi\left(r ; \gamma, E^{\prime}\right)-\psi(r ;-\gamma, E)\right]}{\pi\left(E^{\prime}-E\right)} \rightarrow \\
\rightarrow B(\gamma, E) \delta\left(E-E^{\prime}\right)+B(\gamma, E) \frac{\gamma k}{q|E|} \frac{\cos \left[\psi(r ; \gamma, E)-\psi\left(r ;-\gamma, E^{\prime}\right)\right]}{\pi\left(E-E^{\prime}\right)}, \quad r \rightarrow \infty ; \\
U_{(2)}=U_{(2)}(r ; \gamma, E), \quad 0<\gamma<1 / 2 \\
\left(E-E^{\prime}\right)^{-1} W\left(r ; U_{(2)}, U_{(2)}^{\prime}\right) \rightarrow \\
\rightarrow A(-\gamma, E) \frac{E}{|E|} \frac{\sin \left[\psi(r ;-\gamma, E)-\psi\left(r ;-\gamma, E^{\prime}\right)\right]}{\pi\left(E-E^{\prime}\right)}+\text { БО } \rightarrow \\
\rightarrow A(-\gamma, E) \delta\left(E-U_{(2)}^{\prime}\left(r ; \gamma, E^{\prime}\right), \quad r \rightarrow \infty\right.
\end{gathered}
$$

где

$$
\begin{aligned}
& A(\Upsilon, E)=\Delta^{2}(\Upsilon, E) \frac{2 \pi\left(q_{\mathrm{c} j}+\zeta \Upsilon\right)\left(q_{\mathrm{c} j}|E|+\zeta m \Upsilon\right)}{k q^{2}} \\
& B(\gamma, E)=\frac{|E|}{k} \Delta(\gamma, E) \Delta(-\gamma, E) .
\end{aligned}
$$

Для аналогичных пределов $I_{0}$ получаем соответственно (соотношения справедливы для любых $E, E^{\prime}$ )

$$
\begin{aligned}
& U_{(1)}=U_{(1)}(r ; \gamma, E), \quad U_{(1)}^{\prime}=U_{(1)}\left(r ; \gamma, E^{\prime}\right), \quad \gamma>0, \\
& \left(E-E^{\prime}\right)^{-1} W\left(r ; U_{(1)}, U_{(1)}^{\prime}\right) \rightarrow 0, \quad r \rightarrow 0 \text {; } \\
& U_{(1)}=U_{(1)}(r ; \gamma, E), \quad U_{(2)}^{\prime}=U_{(2)}\left(r ; \gamma, E^{\prime}\right), \quad 0<\gamma<1 / 2 \text {, } \\
& \left(E-E^{\prime}\right)^{-1} W\left(r ; U_{(1)}, U_{(2)}^{\prime}\right) \rightarrow-\frac{2 \gamma}{q\left(E-E^{\prime}\right)}, \quad r \rightarrow 0 ; \\
& U_{(2)}=U_{(2)}(r ; \gamma, E), \quad U_{(1)}^{\prime}=U_{(1)}\left(r ; \gamma, E^{\prime}\right), \quad 0<\gamma<1 / 2 \text {, } \\
& \left(E-E^{\prime}\right)^{-1} W\left(r ; U_{(2)}, U_{(1)}^{\prime}\right) \rightarrow \frac{2 \gamma}{q\left(E-E^{\prime}\right)}, \quad r \rightarrow 0 ; \\
& U_{(2)}=U_{(2)}(r ; \gamma, E), \quad U_{(2)}^{\prime}=U_{(2)}\left(r ; \gamma, E^{\prime}\right), \quad 0<\gamma<1 / 2 \text {, } \\
& \left(E-E^{\prime}\right)^{-1} W\left(r ; U_{(2)}, U_{(2)}^{\prime}\right) \rightarrow 0, \quad r \rightarrow 0 \text {. }
\end{aligned}
$$

Полученные соотношения позволяют вычислить интегралы перекрытия и вывести соотношения ортонормальности для собственных функций радиальных гамильтонианов. В качестве примера рассмотрим вторую некритическую область заряда, другие области заряда, включая критическую и сверхкритическую области, рассматриваются совершенно аналогично.

Мы должны вычислить интеграл

$$
\int_{0}^{\infty} U_{\xi}(r ; E) U_{\xi}\left(r ; E^{\prime}\right) d r,
$$


где $U_{\xi}(r ; E)$ определен формулой (68). Используя соотношения (Б.1)-(Б.3) и (Б.5)(Б.14), находим

$$
\begin{gathered}
\int_{0}^{\infty} U_{\xi}(r ; E) U_{\xi}\left(r ; E^{\prime}\right) d r=C_{\xi}(E) \delta\left(E-E^{\prime}\right), \quad|E|,\left|E^{\prime}\right| \geqslant m, \\
C_{\xi}(E)=A(\gamma, E)+2 \xi B(\gamma, E)+\xi^{2} A(-\gamma, E), \\
\int_{0}^{\infty} U_{\xi}\left(r ; E_{\xi, n}\right) U_{\xi}\left(r ; E^{\prime}\right) d r=0, \quad\left|E^{\prime}\right| \geqslant m, \\
\int_{0}^{\infty} U_{\xi}\left(r ; E_{\xi, n}\right) U_{\xi}\left(r ; E_{\xi, n^{\prime}}\right) d r=0, \quad n \neq n^{\prime} .
\end{gathered}
$$

Нормировочный множитель $A_{n}$ для собственных функций дискретного спектра,

$$
A_{n}^{2}=\int_{0}^{\infty} U_{\xi}^{2}\left(r ; E_{\xi, n}\right) d r
$$

также может быть вычислен, см., например, [14]. Интересно отметить, что мы можем явно проверить выполнение соотношения $A_{n}=Q_{\xi, n}^{-1}$. Для этой цели рассмотрим интеграл

$$
\int_{0}^{\infty} U_{\xi}\left(r ; E_{\xi, n}\right) V\left(r ; E^{\prime}\right) d r=\lim _{r \rightarrow 0} \frac{\operatorname{Wr}\left(r ; U_{\xi}, V^{\prime}\right)}{E^{\prime}-E_{\xi, n}}, \quad V^{\prime}=V\left(r ; E^{\prime}\right), \quad\left|E^{\prime}\right|<m,
$$

где $V(r ; E)$ определяется выражением (69). Используя соотношения (Б.11)-(Б.14), находим

$$
\int_{0}^{\infty} U_{\xi}\left(r ; E_{\xi, n}\right) V\left(r ; E^{\prime}\right) d r=\frac{\omega_{\xi}\left(E^{\prime}\right)}{E_{\xi, n}-E^{\prime}} .
$$

Вспоминая, что $V\left(r ; E_{\xi, n}\right)=U_{\xi}\left(r ; E_{\xi, n}\right)$, получаем окончательно

$$
\int_{0}^{\infty} U_{\xi}^{2}\left(r ; E_{\xi, n}\right) d r=\lim _{E^{\prime} \rightarrow E_{\xi, n}} \frac{\omega_{\xi}\left(E^{\prime}\right)}{E_{\xi, n}-E^{\prime}}=Q_{\xi, n}^{-2} .
$$

Отметим также, что из формул обращения следует равенство $C_{\xi}(E)=Q_{\xi}^{-2}(E)$.

Благодарности. Д. М. Гитман благодарит Бразильские фонды FAPESP и CNPq за постоянную поддержку; Б. Л. Воронов благодарит Бразильский фонд FAPESP и РФФИ (грант № 05-02-17471) за поддержку. И. В. Тютин благодарит РФФИ (грант № 05-01-00996). Б. Л. Воронов и И. В. Тютин также благодарят Программу поддержки ведущих научных школ (грант № НШ-4401.2006.2) за частичную поддержку.

\section{Список литературы}

[1] С. Швебер, Введение в релятивистскую квантовую теорию поля, ИЛ, М., 1963.

[2] V. G. Bagrov, D. M. Gitman, Exact solutions of relativistic wave equations, Kluwer, Dordrecht, 1990.

[3] W. H. Furry, Phys. Rev., 81 (1951), 115-124.

[4] S. P. Gavrilov, D. M. Gitman, Int. J. Modern Phys. A, 15 (2000), 4499-4538.

[5] Синхротронное излучение, Сб. статей, ред. А. А. Соколов, И. М. Тернов, Наука, М., 1966; А.А. Соколов, И. М. Тернов, Релятивистский электрон, Наука, М., 1983; И. М. Тернов, В.В. Михайлин, Синхротронное излучение. Теория и эксперимент, Энергоатомиздат, М., 1986. 
[6] В.И. Ритус, Квантовая электродинамика явлений в интенсивном поле, Тр. ФИАН CCCP, 111, 1979, 5-151.

[7] А. И. Никишов, "Роль связи спина и статистики в квантовой электродинамике с внешним полем, порождающим пары”, Проблемы теоретической физики, Наука, М., 1972, 299-305; Квантовая электродинамика явлений в интенсивном поле, Тр. ФИАН CCCP, 111, 1979, 153-271; В. Г. Багров, Д. М. Гитман, Ш. М. Шварцман, ЖЖЭФ, 68 (1975), 395-399.

[8] D. M. Gitman, J. Phys. A, 10 (1977), 2007-2020; Д. М. Гитман, Е. С. Фрадкин, Ш.М. Шварцман, Квантовая электродинамика с нестабильным вакуумом, Наука, M., 1991.

[9] S. P. Gavrilov, D. M. Gitman, A. A. Smirnov, B. L. Voronov, "Dirac fermions in a magneticsolenoid field", Focus on Mathematical Physics Research, ed. C. V. Benton, Nova Science Publishers, N. Y., 2004, 131-168.

[10] J. von Neumann, Mat. Ann., 102 (1929), 49-131; M. H. Stone, "Linear Transformations in Hilbert Space and their Applications to Analysis", Am. Math. Soc., Colloq. Publ., 15, AMS, N. Y., 1932.

[11] Г. Бете, Э. Солпитер, Квантовая механика атомов с одним и двумя электронами, Физматгиз, М., 1960.

[12] P. A. M. Dirac, Proc. Roy. Soc. London A, 117 (1928), 610-624; 118 (1928), 351-361; C. G. Darwin, Proc. Roy. Soc. London A, 118 (1928), 654-680; W. Gordon, Zs. Phys., 48 (1928), 11-14; E. U. Gordon, G. H. Shortley, The Theory of Atomic Spectra, Cambridge Univ. Press, Cambridge, 1935.

[13] M. E. Rose, Relativistic Electron Theory, Wiley, N. Y., 1961.

[14] А. И. Ахиезер, В. Б. Берестецкий, Квантовая электродинамика, Наука, М., 1969.

[15] W. Greiner, B. Müller, J. Rafelski, Quantum Electrodynamics of Strong Fields, Springer, Berlin, 1985.

[16] Я. Б. Зельдович, В. С. Попов, УФН, 105 (1971), 403.

[17] I. Pomeranchuk, Ya. Smorodinsky, J. Phys. USSR, 9 (1945), 97; С. С. Герштейн, Я. Б. Зельдович, ЖЖЭТФ, 57 (1969), 654; Nuovo Cimento Lett., 1 (1969), 835.

[18] Н. И. Ахиезер, И. М. Глазман, Теория линейных операторов в гильбертовом пространстве, Наука, М., 1966; М. А. Наймарк, Линейные дифференциальные операторы, Наука, М., 1969.

[19] А.И. Плеснер, Спектральная теория линейных операторов, Наука, М., 1965.

[20] И.С. Градштейн, И.М. Рыжик, Таблищы интегралов, сумм, рядов и произведений, Наука, М., 1971.

[21] Справочник по специальным функииям с формулами, графиками и математическими таблицами, ред. М. Абрамовиц и И. Стиган, Наука, М., 1979.

[22] B. L. Voronov, D. M. Gitman, I. V. Tyutin, Self-adjoint differential operators associated with self-adjoint differential expressions, quant-ph/0603187. 